Cahiers Charlevoix

Études franco-ontariennes
Cahiers Charlevoix Études franco-ontariennes

or Crevenerix of

\title{
Francophones et Franco-Ontariens dans l'univers agricole canadien, 1851-1911 : perspectives comparatives
}

\section{Fernand Ouellet}

Volume 1, 1995

URI : https://id.erudit.org/iderudit/1039465ar

DOI : https://doi.org/10.7202/1039465ar

Aller au sommaire du numéro

\section{Éditeur(s)}

Société Charlevoix

Presses de l’Université d'Ottawa

\section{ISSN}

1203-4371 (imprimé)

2371-6878 (numérique)

Découvrir la revue

Citer cet article

Ouellet, F. (1995). Francophones et Franco-Ontariens dans l'univers agricole canadien, 1851-1911 : perspectives comparatives. Cahiers Charlevoix, 1,

291-359. https://doi.org/10.7202/1039465ar

\section{Résumé de l'article}

Fernand Ouellet, historien de l'Université York, s'intéresse à la spécificité de l'expérience franco-ontarienne dans l'univers agricole canadien. Son étude fouillée, fruit d'une longue enquête statistique pour les années 1851 à 1911, présente plusieurs aspects de nouveauté : comparative et régionale, elle permet de corriger les erreurs de perspective et d'isoler les données relatives aux concentrations ethniques partout au Canada durant cette période. L'auteur peut ainsi mettre en évidence les disparités régionales canadiennes, puis relever celles qui existent entre le Québec et l'Ontario, et enfin distinguer même les populations françaises et anglaises de l'Ontario. Bien que " préliminaire ", cette recherche oblige le chercheur à conclure que les Franco-Ontariens ont amélioré leur sort en s'établissant dans cette province, car, pourtant moins riches qu'ailleurs en Ontario, leurs terres, comme celles de leurs nouveaux compatriotes, affichaient un meilleur rendement que celles, plus fertiles, de la région de Montréal ; comme quoi la qualité des terres ne peut seule tout expliquer. 


\section{Francophones et Franco-Ontariens DANS L'UNIVERS AGRICOLE CANADIEN, 1851-1911: PERSPECTIVES COMPARATIVES*}

Fernand Ouellet

Département d'histoire Université York, Toronto

* La recherche pour ce texte a reçu l'appui du programme Killam. Cahiers Charlevoix 1, 1995, pp. 291-359. 
SOMMAIRE

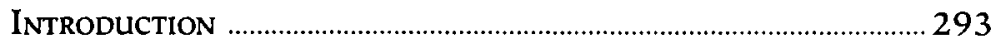

I - Restructuration ou Continuité ..................................................... 296

1. La part du blé dans la récolte: déclin et remontée

2. La production des grains et racines: niveaux et évolution

II - LE CHEPTEL, LE DÉCLIN DE LA PRODUCTION DOMESTIQUE ET L'EMERGENCE DE L'INDUSTRIE LAITIËRE

III - RÉgIONALISATION DE L'AGRICULTURE:

Québec et OntaRio

1. Les disparités régionales au Québec

2. Disparités régionales ontariennes et présence canadienne-française

IV - LES CONCENTRATIONS AGRICOLES FRANCOPHONES

1. La concentration d'Essex-Kent

2. La concentration de Simcoe

3. La concentration de l'Est ontarien

4. La marche vers le Nord

Conclusion 


\section{Francophones et Franco-Ontariens DANS L'UNIVERS AGRICOLE CANADIEN, 1851-1911:}

\section{PERSPECTIVES COMPARATIVES}

\section{INTRODUCTION}

En 1850, le Québec, comme les Maritimes, était devenu un foyer d'intense brassage démographique, surtout pour les Canadiens français. Car les déplacements de population à partir des anciens lieux de peuplement vers les régions périphériques de la province et vers les villes ne suffisaient pas à absorber tous les surplus de population. De ce fait, les migrations vers les États-Unis et les provinces canadiennes situées à l'ouest du Québec étaient une nécessité pour ceux qui, au Québec et dans les Maritimes, devaient trouver du travail ou des terres. Ces mouvements de grande ampleur, qui n'eurent peut-être pas un caractère aussi massif que celui qu'on leur a prêté, eurent cependant des conséquences capitales aussi bien pour ceux qui restèrent que pour ceux qui partirent ${ }^{1}$.

${ }^{1}$ Nous avons tenté, les mettant en rapport les uns avec les autres, de 
En 1851, la population canadienne-française du Haut-Canada se chiffrait à 27079 personnes dont 22725 (83,9 \%) vivaient à la campagne. Ces effectifs étaient modestes comparés à ceux du Québec, le lieu d'origine de la grande majorité de ces individus. À cette date, la population d'origine française s'y élevait à 669228 dont 586870 (88,1\%) habitaient en milieu rural. Soixante ans plus tard, ces rapports avaient été modifiés substantiellement. La migration vers l'ouest avait progressé et l'Ontario était devenu le lieu de résidence de 202387 individus d'origine française dont $110526(54,6 \%)$ étaient des ruraux. Pendant ce temps, la population française du Québec avait plus que doublé, atteignant 1564058 personnes dont 868177 (55,5\%) vivaient en dehors des agglomérations urbaines. Ces effectifs ontariens qui, en 1851 et 1911 , représentaient respectivement $4 \%$ et $12,9 \%$ de ceux du Québec francophone, s'étaient aussi accrus (de $2,9 \%$ à $8 \%$ ) plus rapidement que la population ontarienne.

Il est évident que la fraction substantielle de cette population de langue française vouée à l'agriculture, qu'elle fût originaire du Québec, des Maritimes ou née sur place, entretenait des rapports importants non seulement avec son terroir immédiat mais aussi, quoi que plus indirectement, avec un univers économique et agricole plus vaste, incluant des francophones et des anglophones, qui, de multiples façons, influait sur son revenu et son mode de vie. Pour mieux entrevoir la complexité de ces liens, il aurait peut-être été utile de suivre la voie monographique

\footnotetext{
mesurer ces différents déplacements dans un texte intitulé: «La Colonisation du Saguenay-Lac-Saint-Jean, 1850-1911, en perspective... La marche des francophones dans l'Est du Canada et vers la Nouvelle-Angleterre», Saguenayensia, vol. 36, 1994, pp. 8-27.
} 
en concentrant l'attention sur un minuscule peuplement-témoin en Ontario pour en extraire des éléments de généralisation. Pourtant, une autre démarche sembla davantage susceptible de mettre en valeur le côté exemplaire et la spécificité de l'expérience franco-ontarienne, notamment celle qui consiste à la situer dans le contexte plus large des déplacements et de la concentration en certains lieux de la population canadienne-française adonnée à l'agriculture. D'autant plus que les questions qui préoccupent l'historiographie de l'agriculture se posent à une échelle qui déborde les aires habitées par les Canadiens français et leur confère un sens particulier. Les rapports entre monoculture et agriculture mixte, entre le secteur agricole et les autres secteurs, la diversification de la production, le déclin des productions domestiques, les inégalités parmi les agriculteurs eux-mêmes et la régionalisation à l'intérieur même des provinces, sont autant d'interrogations et de réalités qui invitent aux mises en perspective et aux comparaisons. Il n'est pas question ici de donner une réponse à tout cela mais seulement de faire avancer l'effort de clarification amorcé, en procédant incessamment du général au particulier.

Cette analyse mettra, entre autres, en valeur le fait qu'à l'origine, l'agriculture mixte a partout prévalu sur le territoire canadien actuel, excepté dans le Québec d'avant 1800; que, là seul, le blé y fut la base essentielle de la récolte. Aussi, l'idée de vouloir lier la transition à l'industrie laitière au déclin du blé doit-elle être remise en question. Ce qu'il faut plutôt mettre en valeur à cet égard, ce sont les facteurs: industrialisation et urbanisation. En d'autres mots, la relation entre l'émergence de l'industrie du coton, de la laine et des produits laitiers et le déclin des 
productions domestiques semble beaucoup plus fructueuse. L'analyse mettra aussi en évidence le fait que, là où les francophones étaient ruralement les plus concentrés sur le territoire canadien, non seulement la production agricole y était-elle moins diversifiée mais les niveaux de productions y étaient plus bas. Cette observation vaut aussi à l'intérieur des provinces, y inclus le Québec.

\section{I - Restructuration OU CONTINUITE}

Les historiens ont, semble-t-il, eu tendance à estimer qu'à l'origine les différentes communautés d'agriculteurs ont eu l'habitude au Canada de concentrer leur activité sur une production principale, base de la subsistance et axée potentiellement sur un marché extérieur. Dans leur esprit, il s'agit, bien sûr, du blé qui, lorsque les circonstances devinrent favorables, en vint à jouer le rôle de «staple». Il y a une douzaine d'années, Marvin McGinnis, dans un geste timidement révisionniste visant l'interprétation de John McCallum à propos de l'Ontario, écrivait:

"The apex of the "wheat staple" in eastern Canada was reached in the mid-1850s ». Et il ajoutait: «The main part of the shift to mixed farming had been accomplished by 1870 , well before the settlement of the Canadian West ${ }^{2} »$. Plus récemment encore, comparant en 1991 l'Ontario des origines avec la Nouvelle-France, nous écrivions:

Wheat played a different role in the settlement of Upper

Canada of course. There, although they occupied land

\footnotetext{
${ }^{2}$ Marvin McGinnis, "The Changing Structure of Canadian Agriculture, 1867-1897», Journal of Economic History, Vol. XLII, 1982, pp.194, 192. Voir John McCallum, Agriculture and Economic Development in Quebec and Ontario until 1870, (Toronto, UTP, 1980), pp. 8-24.
} 
better adapted for wheat production, colonists depended upon a mixed agriculture, harvesting a wide variety of grains and other crops. They increased the percentage of wheat in their harvest only as transportation obstacles on the St. Lawrence were removed ${ }^{3}$.

Cette vision fut confirmée deux ans plus tard dans le livre de Douglas McCalla: «In terms of what farmers actually did, however, Upper Canadian agriculture was always a mixed farming system, with wheat as a substantial element in much of the province ${ }^{4} »$.

\section{LA PART DU BLÉ DANS LA RÉCOLTE: DÉCLIN ET REMONTÉE}

En vérité, il n'y eut sur le territoire canadien actuel, que la Nouvelle-France dont l'économie agricole fût érigée principalement au départ sur le blé. En effet, du XVII ${ }^{e}$ au début du XIX ${ }^{e}$ siècle, cette denrée occupa constamment dans les trois régions du Québec où, pourtant, la qualité des sols différait substantiellement de l'une à l'autre, plus des deux tiers, souvent les trois quarts, de la récolte de grains et de racines. L'avoine et les pois constituaient des cultures essentielles mais quantitativement marginales. Avec le temps, le blé local, un des fondements principaux de l'alimentation des habitants, un des principaux moyens de payer les redevances seigneuriales, les dîmes et autres obligations à l'endroit du clergé et des marchands, trouva un marché à l'extérieur et, dès lors, joua d'une certaine façon le rôle de «staple ${ }^{5} »$.

\footnotetext{
${ }^{3}$ F. Ouellet, Economy, Class and Nation in Quebec. Interpretive Essays, (Toronto, Copp Clark Pitman, 1991), p. 133.

${ }^{4}$ Douglas McCalla, Planting the Province. The Economic History of Upper Canada, 1784-1870, (Toronto, UTP, 1993), p. 89.

${ }^{5}$ Voir Ouellet, Economy, Class and Nation, op. cit., Table 8, p. 134; Table 8, p. 180 . Voir aussi chapitre 3 . Ce déclin du blé a été décrit par
} 
Il ne semble pas que, dans les autres colonies de l'Est, le blé ait pu jouer à la même époque un rôle similaire à celui qu'il eut au Québec avant le XIX ${ }^{\mathrm{e}}$ siècle. La pêche étant la grande pourvoyeuse d'aliments et la farine d'importation facile, le profil de cette denrée fut sans doute modeste de sorte qu'une agriculture faite d'éléments divers s'implanta d'abord. Plus tard, même dans les Prairies en voie de naître, bien que le pourcentage de blé, d'avoine et de pommes de terre fût d'abord très élevé dans la moisson (près de $80 \%$ pour les trois), il ne fait pas de doute que l'agriculture y eut un caractère mixte avant 1891 et que les polarisations tenaces autour du blé ne vinrent que plus tard. À ce sujet, notons qu'en 1901, au Manitoba, la défaillance passagère de l'avoine fut compensée par la croissance passagère du seigle qui accapara alors pour cette seule année $32,7 \%$ de la récolte. En Colombie-Britannique, l'agriculture mixte triompha au départ et conserva ce caractère. Ce sont des faits qu'éclaire le tableau 1 qui porte sur cette transition. Cela étant, comment économistes et historiens ont-ils pu associer si intimement le déclin du blé et l'émergence de l'industrie laitière?

Comment peut-on aussi, dans ces conditions, discourir sur le sens du déclin du blé en assumant, simplement et sans plus ample vérification, l'existence universelle à l'origine d'une économie agricole axée principalement sur cette culture? Il semble bien qu'à cet égard, le Québec d'avant 1800 fut unique. C'est d'ailleurs en raison de la force de cette tradition remontant à l'ancienne France que la restructuration

Serge Courville et Normand Séguin presque dans les mêmes termes que nous le faisons dans les textes anciens et récents qui sont reproduits dans cette collection d'essais. Voir Courville-Séguin, Le Monde rural québécois au XIX'e siècle, (SHC, Ottawa, 1989), p. 10s. 
TABLEAU 1

La part des trois denrées (boisseaux) les plus courantes dans la récolte des régions de l'Ouest, 1881-1911

(en pourcentages)

\begin{tabular}{lrrrr}
\hline Manitoba & blé & avoine & pomme de terre total \\
1881 & & & & \\
1891 & 31,0 & 38,2 & 16,7 & 85,9 \\
1901 & 57,0 & 29,6 & 6,2 & 92,8 \\
1911 & 53,5 & 1,7 & 5,5 & 60,7 \\
Territoires* & 46,0 & 40,9 & 3,9 & 90,8 \\
1881 & & & & \\
1891 & 35,3 & 17,7 & 26,3 & 79,3 \\
1901 & 41,7 & 35,3 & 11,7 & 88,7 \\
1911 & 25,2 & 56,2 & 1,2 & 82,6 \\
Colombie-Britannique & 47,2 & 47,1 & 3,4 & 97,7 \\
1881 & & & & \\
1891 & 12,5 & 18,3 & 34,2 & 65,0 \\
1901 & 14,3 & 34,7 & 25,3 & 74,3 \\
1911 & 10,1 & 40,7 & 26,5 & 77,3 \\
& 5,1 & 43,5 & 40,2 & 88,9 \\
\hline
\end{tabular}

SOURCES: Recensements du Canada.

* Par Territoires, nous entendons ici surtout la Saskatchewan et l'Alberta.

relativement rapide au $\mathrm{XIX}^{\mathrm{e}}$ siècle de l'activité agricole autour de l'avoine et de la pomme de terre y eut une signification qu'elle n'eut pas ailleurs. En 1831, le Bas-Canada était devenu pour de bon un gros importateur de blé et de farine. Notons que le déclin du blé, qui n'est pas seulement relatif mais absolu, aurait été encore plus radical dans cette province si les défricheurs des régions périphériques ne lui avaient accordé autant d'importance. Ainsi, en 1881 , $45,5 \%$ du blé produit dans la grande région de Québec l'était dans les endroits où les terres étaient de colonisation récente. Le tableau 2 traduit bien l'ampleur et le caractère irréversible de ce phénomène. Il faut cependant tenir compte du fait que les chiffres de 
1844 à propos du blé et ceux de 1851 relatifs à la pomme de terre reflètent pour une bonne part les répercussions de la mouche à blé après 1831 , d'une part, et celles de la maladie de la pomme de terre de 1846 , de l'autre.

TABLEAU 2

La part des trois denrées (en boisseaux) les plus courantes dans la récolte de grains et racines au Québec, 1827-1911

(en pourcentages)

\begin{tabular}{lrrcc}
\hline & blé & avoine & pomme de terre & total \\
1827 & 22,5 & 18,8 & 52,3 & 93,6 \\
1831 & 22,0 & 27,1 & 46,0 & 95,1 \\
1844 & 4,3 & 33,0 & 45,2 & 82,5 \\
1851 & 15,3 & 44,9 & 22,1 & 82,2 \\
1861 & 6,6 & 43,5 & 31,7 & 81,8 \\
1871 & 4,6 & 34,4 & 41,1 & 80,1 \\
1881 & 4,2 & 41,6 & 30,9 & 76,7 \\
1891 & 3,7 & 39,8 & 35,4 & 78,9 \\
1901 & 3,0 & 50,0 & 24,6 & 77,6 \\
1911 & 1,6 & 56,8 & 26,8 & 85,2 \\
\hline
\end{tabular}

SOURCES: Recensements du Canada.

Il est évident, étant donnée la faible marge laissée à la diversification de la production, que l'implantation de l'agriculture mixte au Québec rencontra des obstacles jusqu'à la fin du siècle. Ainsi, le retour à la pomme de terre de 1861 à 1871 était certainement le signe d'un malaise. D'autant plus que la culture des navets, tellement importante ailleurs, ne reçut qu'une infime attention des producteurs francophones. En 1851, 41,4\% de la production de cette denrée venait de comtés à forte population anglophone. Un demi-siècle plus tard, ce pourcentage était encore de $34,4 \%$. On pourrait même avoir l'impres- 
sion que les agricultuteurs canadiens-français étaient encore à la recherche d'une production dominante susceptible de remplacer le blé. Vers la fin de la période, toutefois, l'avoine paraît avoir commencé à jouer ce rôle. De ce point de vue, le Québec agricole pouvait fraterniser avec certaines parties des Maritimes.

Il est vrai qu'après 1881 les cultivateurs des Maritimes disposaient d'une marge de manœuvre un peu plus considérable que ceux du Québec pour la diversification de la production des grains et racines. N'empêche que les deux agricultures paraissent avoir souffert des mêmes rigidités. Non seulement oscillaient-elles entre l'avoine et la pomme de terre, le blé restant comme autrefois marginal, mais ces produits occupaient une telle place dans la récolte qu'il est même difficile d'entrevoir à cette date un rôle particulièrement dominant pour la pomme de terre à cet endroit. Le tableau 3 le démontre.

\section{TABLEAU 3}

La part des trois denrées (en boisseaux) les plus courantes dans la récolte des Maritimes*, 1871-1911

(en pourcentages)

\begin{tabular}{lcccc}
\hline & blé & avoine & pomme de terre & total \\
1871 & & & & \\
1881 & 2,4 & 29,3 & 54,3 & 86,0 \\
1891 & 4,3 & 23,5 & 55,8 & 83,6 \\
1901 & 3,1 & 23,5 & 53,3 & 79,9 \\
1911 & 3,4 & 31,3 & 37,0 & 71,7 \\
\hline
\end{tabular}

SOURCES: Recensements du Canada.

*île-du-Prince-Édouard, Nouvelle-Écosse et Nouveau-Brunswick. En 1869 , la pomme de terre occupait $88,8 \%$ de la récolte à Terre-Neuve. 
L'Ontario, au contraire, fut une terre de prédilection pour le développement d'une agriculture mixte. Après le premier établissement du Haut-Canada, l'accroissement de la production de blé alla de pair avec l'augmentation de la population, le recul du blé dans le Bas-Canada et les possibilités d'accès au marché extérieur. Donc, des années 1780 à 1850 , surtout à la suite de l'ouverture des canaux, la production s'accrut et continua de le faire jusqu'en 1881. À tel point que le niveau de 1901 était encore presqu'égal au maximum de 1881 , dépassant même la production du Manitoba, de la Saskatchewan et de l'Alberta réunis: 26822242 boisseaux contre 21068823 . En fait, cette province en récoltait encore plus que toutes les autres provinces canadiennes ensemble: 74,6 fois la Colombie-Britannique, 19,6 fois les Maritimes et 12,3 fois le Québec. Ce fait est corroboré autrement par William L. Marr qui conclut:

Perhaps the most dramatic event of the first two decades of the twentieth century was the geographical shift of the centre of production, population, and labour force towards the prairie provinces... The locus of wheat production in Canada moved as dramatically. In 189152,5 per cent of total Canadian acres in wheat production were located in Ontario ${ }^{6}$.

Pendant nombre de décennies, le blé fut peut-être la grande denrée d'exportation mais, quantitativement parlant, son règne en tant que production principale dans un système mixte fut de plus courte durée encore: un peu plus d'une décennie, peut-être, avant que ne s'enracine sa chute progressive. En 1842, la production de blé venait loin derrière celles de l'avoine

\footnotetext{
${ }^{6}$ William L. Marr, «The Wheat Economy in Reverse: Ontario's Wheat Production, 1887-1917», Canadian Journal of Economics, XIV, 1981, p. 136.
} 
et des pommes de terre: le blé $16,4 \%$ et les deux autres $24,4 \%$ et $41,1 \%$, respectivement, de la récolte de grains et racines. En 1848, cependant, suite à la maladie de la pomme de terre, le blé venait en tête avec $31,9 \%$ de la moisson. Le recensement de 1851 reflétait toujours cette situation.

TABLEAU 4

La part des trots denrées (en boisseaux) les plus courantes dans la récolte de gralns et racines de l'Ontarlo, 1851-1911

(en pourcentages)

\begin{tabular}{lrrcc}
\hline & blé & avoine & pomme de terre & total \\
1851 & 32,8 & 29,5 & 13,2 & 75,5 \\
1861 & 24,9 & 20,9 & 13,3 & 59,1 \\
1871 & 14,0 & 21,6 & 16,9 & 52,5 \\
1881 & 16,7 & 24,6 & 11,5 & 52,8 \\
1891 & 12,8 & 28,3 & 10,6 & 51,7 \\
1901 & 10,3 & 33,0 & 7,2 & 50,5 \\
1911 & 10,2 & 44,9 & 8,9 & 64,0 \\
\hline
\end{tabular}

SOURCES: Recensements du Canada.

Pourtant, ce qui est le plus frappant en ce qui concerne l'Ontario, une fois qu'il est tenu compte du poids de ces denrées dans la moisson, c'est l'importance de l'espace laissé à la diversification et, plus spécifiquement, à des productions telles que les navets (parfois au premier rang et, le plus souvent, au second derrière l'avoine), l'orge, les pois et le maïs. En effet, alors que partout ailleurs ces trois productions occupent presque toujours entre $75 \%$ et $95 \%$ de la récolte, leur total se situe le plus souvent en Ontario entre $50 \%$ et $60 \%$.

Ainsi, le déclin du blé au Québec, pour des raisons spécifiques, sa marginalité traditionnelle dans les 
Maritimes, sa chute relative après 1851 , absolue après 1881 , en Ontario, ne sont que des facettes d'un phénomène général qui s'exprime par la progression de l'agriculture mixte sur le territoire. Ce processus touche les Canadiens français du Québec et des Maritimes et fait partie de l'héritage de ceux qui émigrèrent en Ontario et dans les provinces de l'ouest. Derrière un mouvement d'une telle ampleur et universalité, quoique plein de nuances si on le regarde au plan local et régional, il n'est pas possible de ne pas reconnaître l'existence de forces en interaction, commandées de l'intérieur et de l'extérieur, agissant partout sur le territoire. Les contraintes de la tradition, de la qualité des terres, de l'accessibilité et de la raréfaction des terroirs, de la pauvreté, du manque d'instruction, de la compétition, des marchés, des prix et des transports réduisent, orientent les choix et, conséquemment, pèsent plus lourdement sur certains groupes que sur d'autres. Il serait donc trop simple de vouloir tout placer sous le signe de la rationalité quand l'action des individus est sujette à tellement de déterminismes.

\section{LA PRODUCTION DES GRAINS ET RACINES: NIVEAUX ET EVOLUTION}

Ainsi, dans toutes les parties du Canada actuel, y inclus Terre-Neuve, l'agriculture mixte était là en 1830 et se diversifia par la suite. Là où les Canadiens français étaient engagés dans cette activité, ils participèrent aussi à cette orientation. N'empêche que, derrière cette similitude structurelle, on trouve, d'une province à l'autre et à l'intérieur de chacune d'elles, d'énormes inégalités quant aux niveaux de produc- 
tion et à leur évolution. Pour bien marquer le contexte dans lequel s'inscrit notre analyse à ces divers points de vue, il est crucial d'insister sur le fait que la géographie du monde rural canadien-français ne correspondait pas, en général, à celle des hauts niveaux de production.

Ainsi, en $1881,52,5 \%$ de la population rurale canadienne était concentrée dans le Québec et les Maritimes; mais 92,5\% de la population rurale francophone s'y trouvait. Sur ce territoire, la production de grains et racines était de 52,2 boisseaux par campagnard contre 104,9 à l'ouest du Québec. En 1911 , $42,6 \%$ des ruraux canadiens vivaient au Québec et dans les Maritimes mais ceux d'origine française $y$ résidaient dans une proportion de $85,4 \%$ et leur production par campagnard y était de 60,2 boisseaux contre 191 dans la zone Ontario-Ouest.

De telles inégalités existaient à l'intérieur des Maritimes elles-mêmes où, de 1881 à 1911 , avait lieu un déplacement de la population rurale et totale canadienne-française et de la population rurale en général vers le Nouveau-Brunswick. En 1881, 52,3\% des ruraux d'origine française de la région vivaient dans cette province; en 1911, ce pourcentage atteignit $62,8 \%$. Pour la population totale de même origine, ces pourcentages respectifs étaient de $52,1 \%$ et $60,3 \%$. Il est vrai que cette attraction se fit sentir sur les ruraux de toutes origines mais ceux-ci y furent beaucoup moins sujets que les francophones. En $1881,35,5 \%$ des ruraux de la région habitaient dans cette province; quarante ans plus tard, le gain n'avait été que de $4,1 \%$. Ces migrations internes doublées d'émigration s'expliquent pour une bonne part par la situation défavorable de l'agriculture de la 
Nouvelle-Écosse. En 1881, dans cette province, la production par campagnard n'y était que 31,1 boisseaux et ne s'éleva que de 2,2 boisseaux jusqu'en 1911. Le tableau 5 illustre cette façon de voir. Notons, cependant, que les Canadiens français ruraux de cette province s'accommodèrent beaucoup mieux de la situation que les autres: leur proportion de la population en milieu rural passant, pendant ces années, de $10,7 \%$ à $13 \%$.

TABLEAU 5

Production de grains et racines par occupant en Nouvelle-Écosse, 1851-1911 (en boisseaux)

\begin{tabular}{lrrrrrr}
\hline & 1851 & 1871 & 1881 & 1891 & 1901 & 1911 \\
grains et racines & 147 & 199 & 267 & 145 & 168 & 190 \\
blé & 9 & 5 & 9 & 3 & 4 & 4 \\
avoine & 44 & 47 & 89 & 26 & 42 & 55 \\
pommes de terre & 63 & 120 & 132 & 85 & 76 & 66 \\
\hline
\end{tabular}

SOURCES: Recensements du Canada.

Ces chiffres confirment jusqu'à un certain point la vision, dite pessimiste, de Julian Gwyn et de Fazley Siddiq qui insistent, pour la période 1851 à 1871 , non seulement sur l'exceptionnelle et croissante pauvreté de la majorité des agriculteurs de cette province mais sur le développement d'énormes inégalités entre eux et le reste de la population, $y$ compris les femmes célibataires ${ }^{7}$. Notons que, dans ce texte, il n'est pas question de disparités parmi les producteurs agricoles eux-mêmes. Cette interprétation vient d'être remise en question par Kris Inwood et Phyllis Wagg

${ }^{7}$ Julian Gwyn and Fazley Siddiq, «Wealth Distribution in Nova Scotia during the Confederation Era», Canadian Historical Review, LXXIII, 1992, pp. 435-452. 
qui, à notre avis, n'ont fait qu'en pondérer l'aspect un peu trop dramatique. Pas plus que leurs prédécesseurs, ils ne touchent aux inégalités parmi les fermiers eux-mêmes, autrement qu'en fonction de leur âge ${ }^{8}$.

En tout cas, comparé à celui de la Nouvelle-Écosse, l'univers agricole de l'île-du-Prince-Édouard faisait figure de paradis: 124 boisseaux par campagnard en 1881 et 164,4 quarante ans plus tard. Pourtant, ce ne fut pas vers l'île, travaillée par la rareté des terres, la saturation démographique, les forces de la concentration et le faible développement industriel, que se dirigèrent les émigrants canadiens-français de la Nouvelle-Écosse. En terme démographique, l'île était en perte de vitesse dans la région tant pour les Canadiens français que pour les autres. Mais, sur l'île même, ils tinrent mieux le coup que les autres. De 1881 à 1911 , la population de l'île déclina mais leur pourcentage dans la population s'accrut de $9,9 \%$ à $14 \%$ et de $11 \%$ à $14,6 \%$ en milieu rural. Le tableau 6 marque bien le contraste énorme qui existait entre elle et la Nouvelle-Écosse.

TABLEAU 6

Production de grains et racines par occupant sur l'île-du-PrinceEdouard, 1881-1911 (en boisseaux)

\begin{tabular}{lrrrr}
\hline & 1881 & 1891 & 1901 & 1911 \\
grains et racines & 850 & 849 & 1039 & 901 \\
blé & 40 & 41 & 53 & 35 \\
avoine & 260 & 193 & 325 & 282 \\
pommes de terre & 443 & 467 & 354 & 292 \\
\hline
\end{tabular}

SOURCES: Recensements du Canada.

${ }^{8}$ Kris Inwood and Phyllis Wang, «Notes and Comments. Wealth and Prosperity in Nova Scotian Agriculture, 1851-1871», Canadian Historical Review, LXXV, 1994, pp. 239-264. 
Le Nouveau-Brunswick, essentiellement le Nord où $97 \%$ des francophones de la province étaient alors concentrés, fut donc un centre d'attraction pour les habitants d'origine française. Culturellement, l'endroit était certes attrayant mais la province, en plus d'avoir des terres disponibles, était un des hauts lieux de l'exploitation forestière. Ainsi, de 1881 à 1911, l'accroissement de leur pourcentage dans les campagnes y fut plus considérable que dans la population en général: de $21,4 \%$ à $33,4 \%$ en milieu rural et de $17,6 \%$ à $28 \%$ à l'échelle de la province. D'ailleurs, dans cette province, les niveaux de production agricole étaient substantiellement plus élevés que ceux de la Nouvelle-Écosse: 52,6 boisseaux de grains et racines par campagnard en 1881 et 58 en 1911 . Bien que plus bas dans les comtés à grande concentration francophone, 49,1 boisseaux en 1881 et 49,4 en 1911, ces performances n'étaient pas trop éloignées de celles des Québécois, dont le niveau se situait à 54,1 et 55,5 boisseaux respectivement à ces deux dates.

TABLEAU 7

Production de grains et racines par occupant au Nouveau-

Brunswick, 1871-1911 (en boisseaux)

\begin{tabular}{lrrrrr}
\hline & 1871 & 1881 & 1891 & 1901 & 1911 \\
grains et racines & 389 & 371 & 273 & 360 & 383 \\
blé & 7 & 14 & 5 & 10 & 5 \\
avoine & 98 & 89 & 80 & 131 & 145 \\
pommes de terre & 210 & 189 & 127 & 125 & 137 \\
\hline
\end{tabular}

SOURCES: Recensements du Canada.

Très loin derrière l'île-du-Prince-Édouard mais dominant de beaucoup la Nouvelle-Écosse pour la 
production des grains et racines, calculée soit par occupant ou par cultivateur ${ }^{9}$, le Québec était à peu près à égalité avec le Nouveau-Brunswick à cet égard. C'est d'ailleurs dans ces deux provinces, en 1911, où résidaient $30,8 \%$ de la population canadienne et $82,6 \%$ des Canadiens français.

\section{TABLEAU 8}

Production de grains et racines par occupant au Québec, 1831-1911 (en boisseaux)*

\begin{tabular}{lccccc}
\hline & blé & orge & avoine & seigle & pois \\
1831 & 68 & 8 & 84 & 5 & 18 \\
1844 & 8 & 11 & 70 & 3 & 12 \\
1851 & 35 & 6 & 104 & 4 & 16 \\
1861 & 28 & 15 & 184 & 8 & 28 \\
1871 & 16 & 13 & 123 & 4 & 18 \\
1881 & 16 & 10 & 161 & 3 & 34 \\
1891 & 10 & 9 & 107 & 1 & 11 \\
1901 & 14 & 19 & 242 & 1 & 6 \\
1911 & 6 & 16 & 226 & 1 & \\
\multicolumn{5}{c}{} & \multicolumn{5}{c}{16} & & & \\
1831 & sarrasin & mais & pomme de terre & navets & grains** \\
1844 & 2 & 7 & 142 & & 309 \\
1851 & 4 & 1 & 96 & & 212 \\
1861 & 6 & 5 & 51 & 4 & 231 \\
1871 & 13 & 3 & 134 & 9 & 422 \\
1881 & 13 & 5 & 146 & 11 & 356 \\
1891 & 16 & 7 & 119 & 21 & 392 \\
1901 & 13 & 5 & 95 & 16 & 268 \\
1911 & 14 & 10 & 119 & 50 & 475 \\
& 16 & 4 & 107 & 22 & 398 \\
\hline
\end{tabular}

SOURCES: Recensements du Canada.

* Les minots ont été convertis en boisseaux à raison de 1,107 boisseaux pour un minot. Cette façon uniforme de procéder pour tout le territoire québécois se trouve à élever jusqu'à un certain point les performances de la province.

** Grains inclut icl racines.

9 L'utilisation du nombre d'occupants ou du nombre de cultivateurs pour mesurer la production moyenne pose certainement de nombreux 
Comme la plupart de ceux qui allaient s'établir dans les régions périphériques du Québec étaient à la recherche de terres ou en quête d'emplois en attendant des terres, ils ne pouvaient qu'améliorer leur sort lorsqu'ils pouvaient en obtenir. En général, une fois établis, leur situation sur ces nouveaux terroirs n'était pas, pour autant, tout à fait comparable à celle de ceux qui étaient restés dans leur paroisse et avaient continué à pratiquer l'agriculture. De 1851 à 1901, l'écart entre les premiers et les seconds, bien que variable d'un endroit à l'autre, avait certainement diminué; mais il n'était pas disparu. Car, au début du siècle, les premiers produisaient encore environ $15 \%$ de grains et racines de moins que les autres.

Ces chiffres sont si modestes qu'ils ne permettent pas d'insister, autant que le font Courville et Séguin, sur les ajustements rapides de l'ensemble des agriculteurs à l'ouverture de marchés extérieurs et aux besoins d'une économie en voie d'industrialisation. Il est vrai que la hausse considérable de la production du foin confirme cette vision des choses. Il en est ainsi de la croissance spectaculaire des récoltes de tabac au nord de Montréal. Il est, néanmoins, essentiel de constater qu'à part les années 1850 et les deux premières décennies du siècle, il ne se passe rien de vraiment spectaculaire en ce domaine, si ce n'est à propos de l'avoine à ces deux moments. Pour le reste, on serait d'autant plus justifié de parler de stagnation

problèmes qui ne sont pas aussi considérables qu'on pourrait le croire. Mais qu'on utilise l'un ou l'autre indicateur ne modifie pas d'une façon significative les résultats. Notre utilisation de la population comme élément de référence ne fait que confirmer ce que nous savons par ailleurs. Pour une critique de la variable "occupants», voir Normand Fortier, «Les recensements canadiens et l'étude de l'agriculture québécoise", Histoire sociale /Social History, vol. XVII, 1984, pp. 257-286. 
pour la période intermédiaire que "ce système de production [...] laisse, disent Courville et Séguin, une large part aux grandes cultures[... $]^{10} \%$. Pour mieux vérifier cette impression de stagnation ou de restructuration difficile laissée par les données des tableaux 2 et 8 , nous avons préparé un tableau mettant en rapport l'évolution de la production de ces denrées et de la population au Québec, comparant globalement, dans une dernière colonne, celui-ci à l'Ontario?

TABLEAU 9

Production de grains et racines au Québec par rapport à la population, 1831-1911 (en boisseaux per capita)*

\begin{tabular}{rrrrrc}
\hline & blé & \multicolumn{3}{c}{ avoine pommes de terre grains $^{* *}$} & $\begin{array}{c}\text { grains } \\
\text { Québec/Ontario*** }\end{array}$ \\
& & & & & \\
1831 & 6,8 & 8,4 & 14,2 & 30,9 & \\
1844 & 1,5 & 11,5 & 16,0 & 34,8 & \\
1851 & 3,8 & 11,2 & 5,5 & 24,9 & $59,4 \%$ \\
1861 & 2,6 & 17,3 & 12,6 & 39,6 & $56,7 \%$ \\
1871 & 1,9 & 14,0 & 16,7 & 40,7 & $65,2 \%$ \\
1881 & 1,6 & 17,0 & 12,2 & 40,1 & $47,7 \%$ \\
1891 & 1,2 & 12,6 & 11,2 & 31,6 & $40,2 \%$ \\
1901 & 1,3 & 22,4 & 11,0 & 44,0 & $39,0 \%$ \\
1911 & 0.4 & 16.4 & 7.7 & 28.9 & $37.6 \%$ \\
\hline
\end{tabular}

SOURCES: Recensements du Canada.

* Minots convertis en boisseaux à raison de 1,107 par minot.

** Grains inclut ici racines.

*** Grains per capita Québec / grains per capita Ontario, le tout en boisseaux.

Pour mieux comprendre ce tableau, il importe de rappeler qu'en 1734 la récolte de grains et racines de la Nouvelle-France, telle que mesurée par le recensement, se chiffrait à 28,6 boisseaux per capita, dont 21,6 en blé. Cette moyenne de 21,6 se compare

${ }^{10}$ Courville et Séguin, op. cit., p. 11. 
avantageusement avec celle de l'Ontario où, de 1851 à 1901, la production moyenne de froment oscillait entre 10 et 18 minots per capita. Il ne fait pas de doute, puisque les exportations de blé du Québec en vinrent à dépasser les 500000 boisseaux à la veille de la Révolution américaine et le million vers 1800 que la production de blé avait continué à croître pendant la seconde moitié du XVIII ${ }^{e}$ siècle $^{11}$. Cette performance globale de 1734 de 28,6 boisseaux de grains et racines non seulement égalait presque, en volume, celle du Bas-Canada en 1831 mais, puisque constituée de blé dans une proportion de $75,5 \%$, elle la surpassait certainement en valeur. Donc une récolte per capita de 28,6 boisseaux en 1734 , de 30,5 en 1831, de 24,9 vingt ans plus tard, de 31,6 en 1891 et de 28,9 en 1911 laissent peu de doute sur la stagnation prolongée au XIX ${ }^{\mathrm{e}}$ siècle de ce secteur de l'agriculture québécoise.

Ainsi, bien que la prolétarisation des campagnes et la poussée d'émigration vers les États-Unis aient eu des origines multiples et, parfois, contradictoires en apparence, l'idée de stagnation est néanmoins nécessaire à leur compréhension. L'écart considérable qui se creuse alors entre le Québec et l'Ontario en ce qui concerne la production des grains et racines relativement à la population, en est une autre confirmation. Il ne fait pas de doute, en l'occurrence, que, pour ceux qui étaient à la recherche d'emplois ou de terres, l'Ontario et l'Ouest pouvaient constituer des pôles majeurs d'attraction où les immigrants pouvaient espérer une amélioration substantielle de leur sort.

Cette autre comparaison, cette fois Ontario/Québec par occupant, met à nouveau en lumière l'ampleur et

${ }^{11}$ Ouellet, Economy, Class and Nation, op. cit., p. 134s. 
la croissance des inégalités entre les deux provinces et, de cette façon, les avantages qui pouvaient résulter d'un établissement canadien-français en Ontario. Elle prouve cependant que les Canadiens français du Québec n'abusèrent jamais des opportunités qui s'offraient à eux à cet égard. À n'en pas douter, comme ce fut le cas des habitants d'origine française des Maritimes, la décision de quitter le Québec, avec peu d'espoir de retour, était d'abord le fruit de la nécessité et, ensuite, des perspectives de survivance, d'amélioration ou d'accueil dans les lieux de destination. Plus attrayant dans les années 1860-70,

\section{TABLEAU 10}

Production de grains et racines par occupant en Ontario 1851-1911, (en boisseaux)

\begin{tabular}{rrrrrrr}
\hline & blé & orge & avoine & seigle & pois & sarrasin \\
1851 & 127 & 6 & 114 & 5 & 30 & 7 \\
1861 & 195 & 22 & 163 & 8 & 91 & 10 \\
1871 & 83 & 55 & 129 & 3 & 45 & 3 \\
1881 & 134 & 65 & 197 & 8 & 46 & 4 \\
1891 & 95 & 62 & 218 & 5 & 62 & 7 \\
1901 & 122 & 68 & 383 & 9 & 55 & 5 \\
1911 & 89 & 61 & 396 & 5 & & 14
\end{tabular}

maïs pommes de terre navets grains** grains Ont. /Québec***

$\begin{array}{rrrrrr}1851 & 17 & 51 & 49 & 388 & 167,7 \% \\ 1861 & 18 & 104 & 152 & 759 & 179,9 \% \\ 1871 & 2 & 101 & 148 & 597 & 167,3 \% \\ 1881 & 39 & 92 & 197 & 783 & 199,6 \% \\ 1891 & 45 & 82 & 191 & 738 & 274,8 \% \\ 1901 & 94 & 84 & 297 & 1116 & 235,0 \% \\ 1911 & 61 & 76 & 153 & 856 & 215,0 \%\end{array}$

SOURCES: Recensements du Canada.

** Grains inclut ici racines.

*** Grains par occupant: Ontario / grains par occupant: Québec, le tout en boisseaux. 
puisque la population d'origine française y augmenta de $138 \%$, l'Ontario le redevint au cours des deux décennies à la charnière du $\mathrm{XX}^{\mathrm{e}}$ siècle pendant lesquelles cette même population doubla. Â noter aussi qu'en cette fin et début de siècle, alors qu'il y eut ruée générale vers l'Ouest, les effectifs francophones de ces territoires se multiplièrent par six. Évidemment, une portion substantielle de cet accroissement doit être mis au compte de l'immigration.

TABLEAU 11

La production de grains et racines relativement à la population et à la population rurale au Québec, en Ontario et dans l'Ouest 1851-1911 (en boisseaux)

\begin{tabular}{rrrrrrr}
\hline & \multicolumn{2}{c}{ Québec } & \multicolumn{2}{c}{ Ontario } & \multicolumn{2}{c}{ Ouest } \\
& pop. & p.rurale & pop. & p.rurale & pop. & p.rurale \\
& & & & & & \\
1831 & 30,9 & & & & & \\
1844 & 34,9 & & & & & \\
1851 & 24,9 & 29,6 & 41,9 & 48,1 & & \\
1861 & 39,6 & 48,7 & 69,8 & 88,9 & & \\
1871 & 40,7 & 50,9 & 62,4 & 81,2 & & \\
1881 & 40,1 & 54,1 & 84,0 & 115,8 & 29,3 & 29,6 \\
1891 & 31,6 & 46,5 & 78,6 & 125,5 & 112,0 & 152,2 \\
1901 & 44,0 & 73,0 & 112,8 & 193,7 & 82,0 & 114,3 \\
1911 & 28,9 & 55,5 & 76,7 & 158,3 & 139,4 & 229,6 \\
& & & & & & \\
\hline
\end{tabular}

SOURCES: Recensements du Canada.

\section{II - LE CHEPTEL, LE DÉCLIN DE LA PRODUCTION} DOMESTIQUE ET L'EMERGENCE DE L'INDUSTRIE LAITIÈRE

Le développement de l'agriculture, qu'il s'agisse d'un système axé sur une céréale à grande dominance, comme au Québec d'avant 1800, ou d'une agriculture mixte comme cela se pratiqua ailleurs, n'allait pas sans l'existence d'un cheptel, dont la dimension et la composition pouvaient varier dans le temps et 
dans l'espace. Ces changements de direction et écarts territoriaux correspondaient certainement à des vulnérabilités et disponibilités différentes aux circonstances économiques. D'ailleurs, ils étaient d'autant plus prévisibles en l'occurrence que, dans toutes les colonies et provinces après 1850 , les sociétés s'engagèrent, avec des chances inégales et à des rythmes divers, sur la voie de l'urbanisation et de l'industrialisation.

Dans ce contexte favorable à l'expansion des marchés locaux, on s'attendrait à ce qu'au moins dans les deux provinces les plus urbanisées, le Québec et l'Ontario, l'augmentation du nombre d'animaux par ferme fût substantiel. Pourtant, la dimension des cheptels y fut relativement stable. Dans les Maritimes, surtout en Nouvelle-Écosse et à l'île-du-PrinceÉdouard, il y eut, au contraire, déclin alors que, dans l'Ouest en voie de formation accélérée, il y eut plus d'instabilité. Le tableau 12 fait état de cette évolution différencielle.

TABLEAU 12

Évolution de la dimension des cheptels, 1851-1911

(nombre d'animaux par occupant)

N.-É. Î.-P.-É. N.-B. Québec Ontario Ouest Ont/Québec

\begin{tabular}{|c|c|c|c|c|c|c|}
\hline & & & 17,5 & 24,7 & & $141,1 \%$ \\
\hline & & & 19,3 & 26,3 & & $136,3 \%$ \\
\hline 16,7 & & 16,3 & 17,4 & 25,2 & & $144,8 \%$ \\
\hline 14,5 & 24,1 & 14,6 & 17,8 & 21,2 & 21,7 & $119,1 \%$ \\
\hline 12,8 & 21,0 & 13,2 & 13,8 & 21,7 & 26,3 & $157,2 \%$ \\
\hline 12,6 & 22,8 & 14,5 & 18,0 & 26,0 & 30,5 & $144,4 \%$ \\
\hline 11,7 & 20,7 & 13,9 & 20,3 & 26,4 & 19,0 & $129,9 \%$ \\
\hline
\end{tabular}

SOURCES: Recensements du Canada. 
Malgré un certain déclin de la taille de ses cheptels, l'Ille-du-Prince-Édouard faisait quand même, à cet égard comme à celui de la production des grains et racines, figure d'îlot agricole prospère dans les Maritimes. Suffisamment, en tout cas, pour que la Nouvelle-Écosse parût à ses côtés d'une grande pauvreté. Au Nouveau-Brunswick, la taille des cheptels était, vers 1871 , à peine plus considérable qu'en Nouvelle-Écosse, mais on y souffrit moins que dans les deux autres provinces des conditions économiques défavorables. Il est vrai que le Québec surpassait le Nouveau-Brunswick quant au nombre d'animaux par occupant mais il se tenait fort loin derrière l'Ontario et l'Ouest de ce point de vue. Le moins qu'on puisse dire est que, eu égard au nombre d'animaux par occupant, cette infériorité du Québec par rapport aux provinces situées plus à l'ouest, loin de s'estomper avec le temps, se maintint.

Ces évolutions inégales et sans grandes fluctuations, loin de refléter l'idée qu'on se fait d'un changement révolutionnaire, semblent plutôt appartenir à une structure en place depuis longtemps et qui se défait péniblement. Ce diagnostic devient encore plus plausible pour le Québec, si on raccorde le nombre d'animaux per capita pour chaque recensement de 1831 à 1911 à la même série pour la période 1734 à 1831 . Alors, l'idée d'un long déclin des cheptels qui aurait débuté assez tôt au XVIII' ${ }^{e}$ siècle, peutêtre au XVII ${ }^{e}$, et se serait continué jusqu'au $\mathrm{XX}^{\mathrm{e}}$ siècle, surprenante à première vue, pourrait même paraître raisonnable ${ }^{12}$. Mais cela est peu probable

${ }^{12}$ Ibid., tableau 14, p. 141. La population utilisée pour ces tableaux est la population rurale alors que, dans ce texte, il s'agit en plus dans le tableau 13 de la population urbaine. 
puisque, pendant toutes ces années, la population s'est diversifiée quant aux occupations et redistribuée géographiquement selon le mode villes/campagnes. D'ailleurs, un simple coup d'œil sur l'évolution des différentes catégories d'animaux dans les cheptels pendant la même période enlève tout doute quant à l'existence de changements importants. Ainsi, en 1734 , le bétail était numériquement dominé par les bêtes à cornes, alors qu'en 1851 il l'était par les moutons. Un demi-siècle plus tard, les bêtes à cornes occupaient à nouveau le premier rang et les moutons le quatrième. C'est dire que, selon le critière utilisé: le nombre d'animaux par occupant ou per capita et le nombre de bêtes dans chaque catégorie par occupant ou per capita, les chiffres, pris séparément, véhiculent simplement des images apparemment contradictoires d'une réalité complexe.

TABLEAU 13

L'évolution des cheptels au Québec, 1851-1911

(nombre d'animaux per capita)

\begin{tabular}{lcccccc}
\hline & chevaux & vaches & bêtes à cornes & moutons & porcs & total \\
1851 & 0,21 & 0,33 & 0,65 & 0,73 & 0,29 & 1,9 \\
1861 & 0,21 & 0,29 & 0,72 & 0,62 & 0,25 & 1,8 \\
1871 & 0,21 & 0,34 & 0,63 & 0,82 & 0,31 & 2,0 \\
1881 & 0,20 & 0,35 & 0,70 & 0,66 & 0,25 & 1,8 \\
1891 & 0,23 & 0,37 & 0,65 & 0,49 & 0,25 & 1,6 \\
1901 & 0,17 & 0,47 & 0,83 & 0,39 & 0,25 & 1,7 \\
1911 & 0,18 & 0,38 & 0,73 & 0,32 & 0,39 & 1,6 \\
\hline
\end{tabular}

SOURCES: Recensements du Canada.

La stabilité du nombre d'animaux par occupant (tableau 12) se retrouve ici pour le nombre total d'animaux per capita. Pourtant, cet immobilisme 
n'existe plus lorsqu'il s'agit des différentes composantes des cheptels. Ainsi, en 1831, les deux espèces qui regroupaient le plus d'unités étaient, comme autrefois, les bêtes à cornes et les moutons dans une proportion de $69,5 \%$, dont $40,6 \%$ pour les seconds; en 1911, une nouvelle combinaison était apparue bêtes à cornes-porcs avec un pourcentage de 79,1\% des animaux, dont seulement $19,7 \%$ pour les moutons. Par contre l'élevage du cheval était depuis toujours resté stationnaire: un peu plus de $10 \%$ des bêtes. Cela s'explique sans doute par le fait que les agricultureurs québécois, bien davantage que les Ontariens, avaient continué, après 1850 , à utiliser le bouf comme animal de trait.

La chute de l'élevage du mouton, d'une très grande signification au Québec, était, là comme ailleurs, une réaction inévitable à la montée de l'industrie manufacturière qui, après 1871 , entraîna le déclin des industries domestiques, en majeure partie pratiquées par les femmes, souvent avec l'aide des enfants. Cette orientation n'était ni le fruit d'une conspiration anglophone, propriétaires d'une majorité de ces entreprises, ni le résultat d'un complot anti-féminin ${ }^{13}$. La présence sur le marché de cotonnades et de lainages manufacturés à bas prix au Québec et ailleurs avait eu des conséquences négatives sur l'élevage du mouton, la production de la laine locale, peu compétitive, et sur la culture du lin. Dans le cas du mouton, la chose est d'autant plus claire au Québec que cet élevage était moins qu'ailleurs pratiqué par intérêt pour la viande de cet animal que pour sa laine qui servait à fabriquer l'étoffe du pays et la flanelle.

${ }^{13}$ Marjorie Griffin Cohen, Women's Work. Markets and Economic Development in Nineteenth-Century Ontario, (Toronto, UTP, 1988), $257 \mathrm{p}$. 
Notons aussi que la culture du lin, plus importante au Québec qu'ailleurs, avait, depuis toujours, été liée à la fabrication de la toile du pays.

Cette mutation, qui n'est pas un phénomène isolé puisqu'elle s'étendait à la plupart des productions domestiques, n'eut rien à voir avec l'effacement du blé sur le terroir ${ }^{14}$. Pas plus, au reste, que le recul de la production du beurre et du fromage domestiques au profit de celle réalisée en fabrique n'en fut une conséquence. La thèse d'Ankli et Millar sur la transition du blé aux produits laitiers, comme celle de Marvin McGinnis, ne s'applique certainement pas au Québec ${ }^{15}$. Pas plus, d'ailleurs, qu'elle ne vaut ailleurs et, presque certainement pas, pour l'Ontario.

TABLEAU 14

Production de beurre et fromage domestiques et de laine au Québec et en Ontario, 1851-1911 (lbs par occupant)

\begin{tabular}{rrrrrrrr}
\hline & \multicolumn{3}{c}{$\begin{array}{c}\text { Québec } \\
\text { beurre }\end{array}$} & $\begin{array}{c}\text { Ontario } \\
\text { fromage }\end{array}$ & laine & beurre & $\begin{array}{c}\text { fromage } \\
\text { fraine }\end{array}$ \\
1851 & 97,8 & 8,0 & 14,9 & 160,7 & 34,2 & 27,1 \\
1861 & 148,0 & 6,4 & 19,9 & 284,2 & 21,2 & 28,5 \\
1871 & 174,2 & 3,6 & 20,2 & 221,9 & 20,3 & 37,8 \\
1881 & 222,8 & 4,1 & 19,9 & 268,8 & 8,3 & 29,4 \\
1901 & 172,0 & 2,4 & 14,5 & 248,0 & 4,7 & 20,5 \\
1911 & 122,0 & $*$ & 18,5 & 249,9 & $*$ & 22,6 \\
& 122,8 & $*$ & 11,8 & 278,0 & $*$ & 12,1 \\
\hline
\end{tabular}

SOURCES: Recensements du Canada.

* Il existe une production mais tellement infime.

À vrai dire, l'originalité des agriculteurs québécois d'origine française était encore plus évidente dans le

${ }^{14}$ Ouellet, op. cit., p. 132.

${ }^{15}$ Robert E. Ankli and Wendy Millar, «Ontario Agriculture in Transition: The Switch from Wheat to Cheese», Journal of Economic History, vol. XLII, 1982, pp. 207-215. 
domaine de la fabrication des produits laitiers domestiques. Dans leurs familles, dont les racines normandes étaient, pourtant, nombreuses, le lait, bien qu'important dans l'alimentation, était en très grande partie consommé sous forme de breuvage ou avec du pain. La production du beurre était faible et celle du fromage, si elle existait, était minuscule. Ainsi, de 1851 à 1901, les comtés à grande concentration anglophone qui représentaient $20 \%$ de la population produisaient entre $30 \%$ et $40 \%$ du beurre de la province. La prédominance de ces peuplements anglophones, bien que leur rôle à cet égard ait eu tendance à diminuer, était encore plus marquée en ce qui concerne le fromage domestique: $78,5 \%$ de la production en $1851,83,6 \%$ en $1861,69,3 \%$ en 1871 et $52,9 \%$ en 1881 . Si, à ces pourcentages, on ajoute la contribution des producteurs anglophones des cantons inclus dans les comtés massivement francophones, on ne peut qu'être frappé par le caractère spectaculaire du renversement de situation qui s'est produit après 1881 chez les cultivateurs canadiensfrançais, maintenant fortement enclins à la production de beurre et de fromage de fabrique.

Ainsi, à la fin du siècle, l'industrie laitière avait presque donné l'illusion d'avoir remplacé, mais après une longue période d'attente, le blé en tant que production dominante orientée à la fois vers les marchés local et extérieur. En effet, suivant de près l'exemple ontarien, les fabriques de beurre, de beurre et fromage, et de fromage avaient fait leur apparition au Québec après 1860 . Vingt ans plus tard, on en dénombrait 92 sur son territoire et 268 en Ontario. Dès lors, bien que de dimensions plus modestes que ceux de la province voisine, ces établissements se multiplièrent au point qu'en 1901 Québec comptait 1992 
fabriques chez lui contre 1336 en Ontario. De cette façon, les producteurs québécois de lait et de crème, tout en maintenant une certaine production domestique et en ravitaillant les fabriques de beurre et de fromage, furent en mesure de réduire d'une façon radicale l'écart qui les séparait de ceux de l'Ontario.

Il va sans dire que tout cela supposait un accroissement accéléré de l'élevage des vaches laitières. Aussi, leur importance relative parmi les bêtes à cornes s'était accrue substantiellement après 1850 , soit de $50,6 \%$ des têtes de bétail à cette date à $56,2 \%$ en 1901. Par contre, bien que l'industrie laitière ait fait ses premiers pas en Ontario, les vaches laitières y avaient à peine gagné du terrain dans l'ensemble des bêtes à cornes, ne représentant, au début du siècle, que $42,1 \%$ des effectifs de cette catégorie. Il est évident que le producteur ontarien, beaucoup plus que le Québécois, avait aussi misé sur les viandes de boucherie: le bouf et le porc. Une comparaison Ontario / Québec aidera à comprendre leur position respective à chaque moment pour toutes les catégories d'animaux composant le bétail sur les fermes.

\section{TABLEAU 15}

Les animaux de la ferme relativement à la population: une comparaison Ontario/Québec, 1851-1911 (en pourcentages)

\begin{tabular}{lrrcrr}
\hline & chevaux & vaches & bêtes à cornes & moutons & porcs \\
1851 & 104,8 & 97,0 & 121,5 & 141,1 & 213,8 \\
1861 & 128,6 & 110,3 & 101,4 & 135,5 & 212,0 \\
1871 & 142,8 & 117,6 & 138,0 & 114,6 & 174,2 \\
1881 & 150,0 & 117,1 & 127,1 & 106,0 & 148,0 \\
1891 & 156,5 & 110,8 & 140,0 & 97,9 & 212,0 \\
1901 & 188,2 & 100,0 & 134,9 & 120,5 & 280,0 \\
1911 & 172,2 & 107,9 & 113,7 & 87,5 & 194,8
\end{tabular}

SOURCES: Recensements du Canada. 
À propos de ces changements, Marvin McGinnis écrivait:

Cattle, either for beef or for dairy products, were the main substitute for wheat. What has attracted the greatest attention in the literature has been the development of cheese as the "new agricultural staple» of the late nineteenthcentury Canada. Cheese was certainly the most rapidly growing component of agricultural production, especially after 1881 . What must be noted, however, is that cheese never contributed as much to overall agricultural output as butter and was a much smaller component of farm production than beef ${ }^{16}$.

Comme nous l'avons démontré, cette transition n'eut rien à voir avec le blé. Car, dans les Maritimes, le blé fut toujours une denrée marginale et l'agriculture mixte un élément essentiel de la structure. Au Québec, la transition du blé vers l'agriculture mixte eut lieu entre 1800 et 1830 , alors que, dans l'Ouest, l'établissement d'un système mixte précéda la montée du blé. Même en Ontario, terroir propice à cette agriculture mixte, où les choses semblent plus enchevêtrées, le rapport supposé entre le recul du froment et l'émergence de l'industrie laitière n'est pas non plus tellement plausible. Il semble, au contraire, que la chute de l'élevage du mouton, la croissance de l'élevage des vaches laitières et des animaux de boucherie, bœuf et porc, sont des événements qui relèvent d'une conjoncture qui entraîne le recul des productions domestiques et une réorientation de l'élevage. À cet égard, toute référence à l'industrialisation, l'urbanisation et l'ouverture de marchés extérieurs est essentielle.

\footnotetext{
${ }^{16}$ Marvin McGinnis, "The Changing Structure of Canadian Agriculture, 1867-1897», Journal of Economic History, 1982, p. 195s.
} 
La démarche suivie jusqu'à maintenant a done permis, en voyageant d'une province à l'autre vers l'ouest, de dégager non seulement des similarités mais aussi des différences et de grandes inégalités quant aux différents aspects de la production agricole et, il va de soi, au revenu de la terre. Les contrastes qui existaient entre le Québec et les Maritimes, que ce fût au plan des récoltes de grains, de l'élevage et des produits domestiques, se retrouvaient aussi marqués entre le Québec et l'Ontario. Pour mieux comprendre la situation mouvante des éléments francophones, de ceux qui habitaient le Québec et de ceux qui allèrent vivre en Ontario, il est indispensable de pénétrer à l'intérieur des provinces et dans leurs régions. Car, ni au Québec ni en Ontario, ces groupes n'étaient également répartis sur le territoire et également bénéficiaires des avantages qu'offraient les terroirs.

\section{III - Régionalisation de l'agriculture: Québec et Ontario}

L'univers qui fait l'objet de cette analyse était donc, pour une large part, divers et inégal. Il le devenait d'autant plus que le processus de régionalisation suivait son cours. Car, aux régions, correspondaient non seulement des réseaux de solidarités mais aussi des disparités économiques spécifiques. Nous avons déjà dit que la géographie des Canadiens français ne reflétait pas, en général, celle des plus hauts revenus agricoles. La chose devient encore plus évidente lorsqu'il est fait état de la pesanteur agricole relative de chaque région à l'intérieur des provinces. 
1. LES DISPARITÉs RÉGIONALES AU QuÉBEC

Il a déjà été établi que, sur le territoire canadien, les Canadiens français furent, en général, sousreprésentés dans les endroits où les niveaux de production agricole étaient les plus élevés. La chose se vérifie même à l'intérieur du Québec où ils avaient, depuis les débuts de la Nouvelle-France jusqu'à 1784 au moins, été sur-représentés démographiquement en dehors du district de Montréal et sous-représentés dans les mêmes lieux en ce qui regarde la production des grains et racines. Ces faits sont d'autant plus significatifs qu'ils avaient depuis 1760 été plus fortement représentés parmi les ruraux, les travailleurs primaires et, en particulier, les agriculteurs que leurs compatriotes des autres origines. Leur suprématie dans ces domaines s'était affirmée davantage au cours des années 1851-1911 parce que les anglophones avaient été relativement plus nombreux à quitter ces secteurs que les francophones.

Ainsi, en 1732, 63,3\% de la population de la NouvelleFrance était concentrée dans les districts de Québec et de Trois-Rivières mais la paysannerie de ce territoire ne produisait que $55,8 \%$ des grains et racines. En 1765 , ces pourcentages respectifs étaient de $59,2 \%$ et de $51,2 \%^{17}$. Encore, vers 1850 , les mêmes disparités existaient et prévalurent jusqu'à 1911 au moins. Ainsi, en 1851 , seulement $45,3 \%$ de la population de la province résidait dans ces deux zones qui, en gros, recouvraient les anciens districts de Québec et TroisRivières. Mais les habitants d'origine française du Québec y étaient présents dans une proportion de $48,3 \%$. Pour les ruraux, le même contraste existait: $46,3 \%$ des ruraux en général et $48,9 \%$ de ceux d'ori-

${ }^{17}$ Ouellet, op. cit., p. 132. 
gine française de la province habitaient sur ce grand territoire. À cette date, les paysans du lieu ne produisaient que $43,2 \%$ de la récolte de grains et racines.

Ces écarts s'étaient non seulement perpétués jusqu'en 1911 mais ils s'étaient élargis, de sorte que, relativement aux effectifs provinciaux, $43,7 \%$ de la population en général, 58,6\% des ruraux, 50,6\% de la population canadienne-française et $62,8 \%$ des ruraux canadiens-français occupaient maintenant ce territoire. Malgré une remontée subite en 1911 de la production des grains et racines dans le seul district de Québec, la part de cette immense région dans les récoltes n'était que de $48,3 \%$. Notons aussi que, de 1871 à 1901 , le poids démographique relatif de la région de Montréal, celle où l'urbanisation progressait le plus rapidement, déclina. Il faut y voir le résultat de la poussée colonisatrice des zones périphériques des districts de Québec et de Trois-Rivières. Le tableau 16 illustre l'aspect démographique de cette évolution.

TABLEAU 16

Répartition par district* de la population francophone du Québec, 1851-1911 (en pourcentages du Québec)

\begin{tabular}{lcccccc}
\hline & \multicolumn{2}{c}{ Québec } & \multicolumn{2}{c}{ Trois-Rivières } & \multicolumn{2}{c}{ Montréal } \\
& pop. & rurale & pop. & rurale & \multicolumn{2}{c}{ pop. rurale } \\
1851 & 35,3 & 35,1 & 13,0 & 13,8 & 51,7 & 51,1 \\
1861 & 36,1 & 37,3 & 13,5 & 14,7 & 50,4 & 48,0 \\
1871 & 38,6 & 40,0 & 13,3 & 14,5 & 48,1 & 45,5 \\
1881 & 38,3 & 41,4 & 15,8 & 17,2 & 45,9 & 41,4 \\
1901 & 35,8 & 43,8 & 14,7 & 18,4 & 49,5 & 37,8 \\
1911 & 36,9 & 46,7 & 13,7 & 16,1 & 49,4 & 37,1 \\
\hline
\end{tabular}

SOURCES: Recensements du Canada.

* Les frontières des trois districts correspondent en gros à ceux qui existaient à l'époque française. 
Dans le district de Québec, la structure de la production était la même qu'ailleurs. Un système mixte y était aussi apparu plus par la force des choses qu'à la suite de choix délibérés. Une culture, le blé, y déclinait comme ailleurs, bien que tenant mieux le coup, finalement, que dans les deux autres districts. La solution de rechange, l'avoine, ne s'affirma qu'en fin de période, supplantée qu'elle fut jusque-là par la récolte de pommes de terre. En fait, jusqu'en 1891, la pomme de terre y accapara une portion de la récolte qu'elle n'obtint nulle part sur le reste du territoire québécois. En 1871, cette récolte y était de 199 boisseaux par occupant, de 132 dans le district de Trois-Rivières et de 120 dans celui de Montréal. Comme ces trois denrées monopolisaient une énorme proportion de la production des grains et racines, entre $84 \%$ et $92 \%$, il est évident que la diversification des cultures était réduite au minimum comparée à ce qu'elle était à Trois-Rivières (entre $83 \%$ et $77 \%$ ) et à Montréal (entre $80 \%$ et $72 \%$ ). Vue sous l'angle production/population, cette agriculture était celle qui bougea le moins pendant ces soixante années.

Le district de Trois-Rivières constituait, comme autrefois, une zone intermédiaire entre les deux autres. La culture de l'avoine y reçut plus d'attention de la part des cultivateurs de cette région qu'elle n'en reçut de ceux situés plus à l'est. En 1901, la récolte d'avoine par occupant était de 174 boisseaux dans le district de Québec, de 231 dans celui de TroisRivières et de 302 dans la région de Montréal. La diversification de la production, partout pénible, s'y fit mieux qu'à l'est mais moins bien que dans la région de Montréal. Le tableau 17, mis en rapport avec le précédent, éclaire certains aspects de cette question. 
Franco-ONTARIENS DANS L'UNIVERS AGRICOLE

TABLEAU 17

Répartition par district de la production de grains et racines (en boisseaux) au Québec, 1851-1911

(en pourcentages du Québec)

\begin{tabular}{lcccc}
\hline & Québec & Trois-Rivières & Montréal & total \\
1851 & 29,3 & 13,9 & 56,8 & 100 \\
1861 & 28,8 & 14,2 & 57,0 & 100 \\
1871 & 32,4 & 14,4 & 53,2 & 100 \\
1881 & 29,2 & 13,0 & 57,8 & 100 \\
1901 & 29,3 & 14,4 & 56,3 & 100 \\
1911 & 34,1 & 14,2 & 51,7 & 100 \\
\hline
\end{tabular}

SOURCES: Recensements du Canada.

Le district de Montréal, au contraire, était l'endroit où la densité agricole surpassa toujours la densité de la présence francophone, aussi bien en milieu rural qu'urbain. En 1732, déjà, des pourcentages de 39,7 pour la population et de 44,2 pour la production des grains. En $1765,40,8 \%$ pour la population et $47,8 \%$ pour la récolte. Il ne fait pas de doute que ces rapports entre territoire, démographie et production agricole étaient des faits de longue durée. Non seulement avaient-ils survécu à la chute du blé mais ils s'étaient même renforcés avec la montée de l'avoine et de la suprématie de la région de Montréal quant à la croissance industrielle et urbaine.

Ces disparités croissantes entre la région de Montréal et le reste de la province étaient un élément de cette structure cristallisée à l'époque de la NouvelleFrance et embrassant à la fois la production des grains, des racines et l'élevage des animaux. En effet, en $1732,39,7 \%$ de la population habitait ce district mais $45,6 \%$ des animaux s'y trouvaient. Avec le temps, cependant, cet écart avait été réduit. Après 1851 , la tendance inverse, bien que beaucoup moins 
marquée que dans le cas des grains, prévalut jusqu'au début du siècle.

Vue dans le détail, cette évolution apparaît non seulement plus complexe mais plus susceptible de changement. Ainsi, aussi loin qu'on remonte dans le passé, le district de Québec avait été en retard sur les deux autres, plus particulièrement sur Montréal, pour l'élevage du cheval. Il en fut ainsi pour l'élevage du porc. De telles disparités, inexistantes avant 1831 en ce qui concerne les bêtes à cornes, étaient, cependant, apparues en 1851 et prirent de l'ampleur par la suite.

Par contre, ce district semble avoir gagné du terrain dans le domaine de l'élevage du mouton et de la production de la laine domestique. En 1851, ce territoire était, relativement à toutes les catégories de population mentionnées ici, derrière les deux autres districts à ces deux points de vue. Mais, en fin de période, sa contribution au niveau provincial était de $53 \%$ des têtes pour les moutons et de $52,2 \%$ des livres de laine produite. Notons cependant que, même si le nombre de moutons avait décliné après 1871 , la quantité de laine produite avait continué sa lente ascension jusqu'en 1901. En vérité, c'est le moment où, dans le reste de la province après 1871 , il y eut déclin substantiel du nombre de moutons et de la production de la laine. Cette différence reflète certainement le caractère plus traditionnel de l'agriculture et le retard du secteur manufacturier dans cette région.

La même interprétation vaut, avec des nuances, pour l'élevage des vaches laitières et la production du beurre et du fromage domestiques. Pourtant, dans le cas des vaches laitières, l'accroissement du nombre de bêtes par occupant fut inférieur dans le district 
de Québec à ce qu'il était dans les deux autres districts. En 1851 , cette région, où la campagne était canadienne-française dans une proportion de plus de $80 \%$, ne produisait que $20 \%$ du beurre domestique fabriqué dans la province, alors que la part du district de Montréal, avec sa substantielle minorité anglophone, s'élevait à 63,9\%. Mais, par la suite, les cultivateurs du lieu augmentèrent leur production plus rapidement que les autres jusqu'en 1881 et la diminuèrent moins qu'eux jusqu'en 1911. De sorte qu'en fin de période ils fabriquaient $50 \%$ du beurre domestique de la province. La sous-représentation originelle du district de Québec était encore plus énorme quant à la préparation du fromage domestique: seulement $3,8 \%$ de la production en 1851 contre plus de $80 \%$ pour le district de Montréal. En 1911, le pourcentage de sa participation s'était accru mais ne totalisait encore que $6,1 \%$. C'est que le district de Montréal avait non seulement maintenu sa suprématie à cet égard mais avait pris, tout comme la région de Trois-Rivières, une avance substantielle dans l'établissement des fabriques de beurre et de fromage. Le retard significatif de la région de Québec allait presque de soi en l'occurrence.

Il y eut donc au Québec, dans l'ensemble et presque de temps immémorial, une sur-représentation des Canadiens français ou des ruraux canadiensfrançais dans les districts où le revenu agricole était le moins élevé. Ainsi, le contraste entre la région de Montréal, déjà significatif avec celle de TroisRivières, était le plus marqué avec le district de Québec. Naturellement, à l'intérieur de chacun de ces territoires, il y eut aussi des disparités importantes d'un lieu à un autre. N'empêche qu'au niveau de la province, les choses se passèrent, eu égard à la 
situation des francophones dans l'univers agricole, comme elles le faisaient sur l'ensemble du territoire canadien.

\section{Disparités RÉgIONALES ONTARIENNES ET PRÉSENCE CANADIENNE-FRANÇAISE}

Lorsque les Canadiens français poussèrent leur marche vers l'Ontario après 1840 , ils eurent d'abord tendance à se concentrer davantage dans l'Est de la province pour ensuite, dans un second temps, s'avancer vers le Nord. En 1851 , environ 30 \% de la population et de la population rurale de la province habitait l'Est. Mais les Canadiens français de la province s'y trouvaient dans une proportion de $50,9 \%$, alors que, parmi eux, les ruraux de même origine y étaient relativement moins concentrés: $47,9 \%$. Cette région était, d'ailleurs, fortement sous-représentée par rapport au Centre et au Sud-Ouest en ce qui regarde la production des grains: $24,3 \%$ des grains et racines, $14,9 \%$ du blé, $26,2 \%$ de l'avoine, $40 \%$ des pommes de terre et $6,2 \%$ des navets.

En fin de période, le pourcentage de la population de la province habitant l'Est et le Nord était encore de $30 \%$, mais celui de la population rurale s'élevait à $43,5 \%$. De leur côté, les habitants d'origine française y avaient les trois quarts de leurs effectifs. Pourtant, la participation de ces deux régions à la production des grains et racines n'avait pas augmenté au rythme de la croissance démographique. En 1911, leur part de la récolte de la province était de $18,6 \%$ pour les grains et racines, $5,8 \%$ pour le blé, $23,8 \%$ pour l'avoine, $36 \%$ pour les pommes de terre et $7,8 \%$ pour les navets. Il est évident qu'à cette date et à ce niveau régional, les migrants canadiens-français n'occu- 
TABLEAU 18

Évolution de la population ontarienne et canadienne-française par régions, 1851-1911 (en pourcentages de l'Ontario)

\begin{tabular}{|c|c|c|c|c|}
\hline & $(1)^{*}$ & $(2)^{*}$ & $(3)^{*}$ & $(4)^{*}$ \\
\hline Sud-Ouest & & & & \\
\hline 1851 & 20,9 & 28,5 & 22,0 & 31,1 \\
\hline 1861 & 27,1 & 19,5 & 25,3 & 20,5 \\
\hline 1871 & 30,3 & 26,0 & 33,6 & 29,8 \\
\hline 1881 & 32,0 & 24,1 & 34,3 & 27,1 \\
\hline 1901 & 27,2 & 18,1 & 30,6 & 21,6 \\
\hline 1911 & 23,0 & 15,4 & 28,3 & 17,8 \\
\hline \multicolumn{5}{|l|}{ Centre } \\
\hline 1851 & 48,3 & 20,6 & 47,0 & 20,9 \\
\hline 1861 & 46,5 & 14,8 & 46,6 & 15,1 \\
\hline 1871 & 44,3 & 16,7 & 40,9 & 16,7 \\
\hline 1881 & 42,0 & 14,9 & 37,6 & 12,7 \\
\hline 1901 & 43,4 & 11,0 & 35,2 & 8,1 \\
\hline 1911 & 46,4 & 10,2 & 35,7 & 6,9 \\
\hline \multicolumn{5}{|l|}{ Est } \\
\hline 1851 & 30,7 & 50,9 & 31,0 & 47,9 \\
\hline 1861 & 25,9 & 64,7 & 27,6 & 63,4 \\
\hline 1871 & 24,3 & 55,2 & 24,2 & 50,6 \\
\hline 1881 & 23,4 & 58,6 & 24,8 & 56,9 \\
\hline 1901 & 23,2 & 56,9 & 26,5 & 52,2 \\
\hline 1911 & 20,1 & 50,3 & 23,4 & 46,8 \\
\hline \multicolumn{5}{|l|}{ Nord** } \\
\hline 1861 & 0,5 & 0,9 & 0,5 & 0,9 \\
\hline 1871 & 1,0 & 2,1 & 1,3 & 2,8 \\
\hline 1881 & 2,5 & 2,4 & 3,3 & 3,2 \\
\hline 1901 & 6,1 & 14 & 7,7 & 18,0 \\
\hline 1911 & 10,4 & 24 & 12,5 & 27,8 \\
\hline
\end{tabular}

SOURCES: Recensements du Canada.

** Pour cette période, nous avons annexé la Muskoka au Nord.

(1)* égale population totale;

(2)* égale population totale d'origine française;

(3)* égale population rurale totale;

(4)* égale population rurale d'origine française.

paient que secondairement des terres dans les endroits où l'activité agricole était la plus rémunératrice. Leur préférence allait, semble-t-il, vers une pratique 
qui associait agriculture et exploitation forestière. Le tableau 18 illustre ces mouvements de population ${ }^{18}$.

Ces Canadiens français étaient donc concentrés dans les régions où le blé avait la moindre part dans les récoltes et où cette portion congrue déclina le plus rapidement. En 1851, dans l'Est, la production de blé par occupant était un peu plus de la moitié de ce qu'elle était dans le Sud-Ouest et seulement un tiers de ce qu'elle était dans le Centre. En 1911, ces proportions par rapport au Sud-Ouest et au Centre ne dépassaient pas un sixième et un cinquième respectivement. La seule production qui dénotait une sur-représentation constante de l'Est par rapport à la population, était celle de la pomme de terre.

TABLEAU 19

Le poids des régions ontariennes dans la production des grains et racines (en boisseaux), 1851-1911 (en pourcentages de l'Ontario)

\begin{tabular}{rrrrrr}
\hline & Sud-Ouest & Centre & Est & Nord & total \\
1851 & 19,2 & 56,4 & 24,4 & & 100 \\
1861 & 30,7 & 49,7 & 19,5 & & 100 \\
1871 & 30,5 & 53,3 & 15,7 & 0,4 & 99,9 \\
1881 & 34,9 & 46,8 & 17,2 & 1,1 & 100 \\
1901 & 40,1 & 44,1 & 14,1 & 1,7 & 100 \\
1911 & 37,1 & 44,2 & 15,8 & 2,8 & 99,9 \\
\hline
\end{tabular}

SOURCES: Recensements du Canada.

La suprématie du Centre et du Sud-ouest dans la production des grains et racines était donc considé-

\footnotetext{
${ }^{18}$ Pour ces mouvements de population, volr Gaétan Gervals, «L'Ontario françals, 1821-1910" et F. Ouellet, "L'Évolution de la présence francophone en Ontario: une perspective économique et sociale", dans C. Jaenen (éd.), Les Franco-Ontariens, (Ottawa, PUO, 1993), pp. 49-201.
} 
rable. Alors que leur population oscillait, de 1851 à 1911 , entre $69 \%$ et $74 \%$ de celle de la province, leur part dans la production de ces denrées s'échelonnait entre $75 \%$ et $84 \%$. Pour le blé, ces pourcentages variaient entre $85 \%$ et $94 \%$; pour l'avoine, entre $73 \%$ et $78 \%$; pour les navets, entre $92 \%$ et $97 \%$. Pendant ce temps, la population d'origine française y perdait de son importance relativement à l'ensemble des effectifs de même origine dans la province: de $49,1 \%$ en 1851 à $25,6 \%$ soixante ans plus tard.

\section{TABLEAU 20}

Suprématie du Centre et Sud-Ouest: la production du blé, de l'avoine, des navets et des pommes de terre (en boisseaux) 1851-1911 (en pourcentages de l'Ontario)

\begin{tabular}{cccccc}
\hline & blé & avoine & navets & pommes de terre & population \\
1851 & 85,0 & 73,8 & 93,7 & 60,0 & 69,2 \\
1861 & 85,0 & 74,5 & 95,7 & 65,3 & 74,0 \\
1871 & 87,9 & 78,9 & 97,3 & 67,8 & 74.6 \\
1881 & 94,4 & 75,9 & 94,3 & 66,0 & 72,0 \\
1901 & 88,8 & 78,1 & 93,9 & 67,4 & 70,6 \\
1911 & 93,6 & 76,2 & 92,1 & 64,0 & 69,4 \\
\hline
\end{tabular}

SOURCES: Recensements du Canada.

Dans l'Est, une fois tenu compte de la part du blé, de l'avoine et des pommes de terre dans la récolte, il $y$ avait, au contraire, peu de place pour la diversification de la production. À cet égard, cette partie de l'Ontario était certainement avantagée relativement au nord de la province et même par rapport à la région de Montréal. Mais elle était loin derrière le Sud-Ouest et le Centre. Les écarts entre l'Est et les deux régions situées plus à l'ouest étaient moins prononcés en ce qui concerne les animaux de la ferme, continuant, néanmoins, de s'accroître 
jusqu'au début du siècle. Pendant la décennie suivante, la position relative de l'Est s'améliora considérablement.

TABLEAU 21

Répartition des animaux de la ferme en Ontario par régions 1851-1911 (en pourcentages de la province)

\begin{tabular}{lccccc}
\hline & Sud-Ouest & Centre & Est & Nord & total \\
1851 & 23,3 & 46,5 & 30,2 & & 100 \\
1861 & 31,5 & 43,4 & 25,0 & & 100 \\
1871 & 35,7 & 41,5 & 22,5 & & 99,8 \\
1881 & 38,6 & 37,4 & 22,9 & 1,1 & 100 \\
1901 & 36,8 & 37,8 & 21,8 & 3,5 & 99,9 \\
1911 & 37,1 & 35,4 & 23,6 & 3,9 & 100 \\
\hline
\end{tabular}

SOURCES: Recensements du Canada.

Le fait que le Nord, peuplement en voie de naître, était alors universellement sous-représenté, qu'il s'agisse d'élevage de toutes les espèces d'animaux ou des productions domestiques, ne faisait que refléter une grande faiblesse dans la production des grains et racines, moins marquée, toutefois, dans celle de la pomme de terre. Dans l'Est, où l'économie agricole avait atteint une certaine maturité, la situation était beaucoup plus complexe. Aussi, l'examen de l'évolution des cheptels par espèces met à jour des changements majeurs, assez similaires à ceux observés au Québec et dans la région de Montréal, en particulier.

Car, en 1851, les cheptels, comme au Québec, étaient numériquement dominés par les bestiaux et les moutons. Ces espèces regroupaient alors $68,2 \%$ des animaux dans le Sud-Ouest, $66,8 \%$ dans le Centre et 70,5\% dans l'Est. Les moutons étaient partout les plus nombreux: $37,5 \%, 38,3 \%$ et $40,3 \%$ respective- 
ment. En 1911, toujours comme au Québec, cette combinaison avait été transformée, excepté dans le Nord, où l'élevage du mouton avait progressé et celui du porc régressé. Ainsi, les catégories bêtes à cornesporcs regroupaient à cette date $76,4 \%$ des animaux dans le Sud-Ouest, 73,3\% dans le Centre, 75,5\% dans l'Est et 64,5\% dans le Nord. Le gros bétail était, maintenant, le plus nombreux alors que le mouton venait partout, excepté dans l'Est, au dernier rang.

TABLEAU 22

Répartition des animaux de la ferme par espèces dans l'Est de l'Ontarlo, 1851-1911 (en pourcentages)

\begin{tabular}{rrrcrrr}
\hline & chevaux & vaches & b. à cornes* & moutons & porcs & total \\
1851 & 9,0 & 13,8 & 30,2 & 40,3 & 20,5 & 100 \\
1861 & 13,7 & 16,9 & 34,2 & 40,5 & 11,6 & 100 \\
1871 & 11,9 & 19,9 & 36,2 & 38,2 & 13,7 & 100 \\
1881 & 13,3 & 24,5 & 42,5 & 32,6 & 11,6 & 100 \\
1901 & 11,9 & 29,9 & 51,9 & 17,6 & 17,8 & 99,2 \\
1911 & 12,1 & 25,9 & 49,0 & 12,3 & 26,5 & 99,9 \\
\hline
\end{tabular}

SOURCES: Recensements du Canada.

* Vaches aussi incluses parmi les bêtes à cornes.

Dans l'Est, contrairement à ce qui se passait dans les deux autres régions situées plus à l'ouest, l'élevage des chevaux et des porcs était alors, relativement parlant, en régression. Mais le changement le plus important était celui du recul des productions domestiques. À cet égard, la chute de l'élevage du mouton était d'autant plus significative que cette région était celle où cet animal avait occupé le plus de place dans les cheptels. C'est là aussi que le déclin des productions domestiques, non seulement pour la laine, la flanelle et l'étoffe mais pour le beurre et le fromage, fut le plus marqué. Pas étonnant que 
l'élevage des vaches laitières et la production du beurre et du fromage de fabrique aient pris des proportions qui rappellent ce qui se passait au même moment dans la région de Montréal.

TABLEAU 23

Comparaison: région de Montréal/Est de l'Ontario quant au

(1) nombre de boisseaux de grains et racines, (2) nombre

d'animaux et (3) produits domestiques en livres par occupant, 1851-1911 (en pourcentages)*

\begin{tabular}{lrrrrr}
\hline & 1851 & 1861 & 1881 & 1901 & 1911 \\
& & & & & \\
grains et racines & 80,1 & 90,2 & 85,1 & 87,2 & 80,8 \\
blé & 67,1 & 33,3 & 45,4 & 30,9 & 29,0 \\
avoine & 112,2 & 146,8 & 93,4 & 92,6 & 79,9 \\
pommes de terre & 67,0 & 101,2 & 62,5 & 73,6 & 99,0 \\
navets & 8,5 & 31,4 & 48,4 & 58,2 & 61,6 \\
& & & & & \\
animaux & 75,3 & 91,5 & 95,0 & 77,9 & 79,1 \\
chevaux & 109,1 & 96,8 & 92,6 & 69,0 & 87,1 \\
vaches & 100,0 & 89,5 & 83,7 & 79,4 & 76,1 \\
bêtes à cornes & 86,3 & 103,9 & 90,6 & 81,7 & 75,6 \\
moutons & 68,4 & 75,8 & 95,4 & 83,7 & 87,5 \\
porcs & 58,0 & 100,0 & 108,7 & 72,1 & 78,3 \\
& & & & & \\
produits domestiques & & & & & \\
beurre & 57,5 & 72,7 & 81,7 & 65,0 & 55,4 \\
fromage & 43,1 & 65,3 & 8,9 & & \\
laine & 63,1 & 76,1 & 75,7 & 87,8 & 86,0 \\
& & & & & \\
\hline
\end{tabular}

SOURCES: Recensements du Canada.

* Pour le district de Montréal, les minots ont été convertis en boisseaux à raison de 1,107 .

Après ce qui a été dit au sujet du statut d'infériorité agricole de l'Est et du Nord par rapport au Centre et au Sud-Ouest de la province, il serait tentant de prendre pour acquis que les immigrants francophones venus des Maritimes et du Québec, surtout ceux de la région de Montréal, avaient fait un mau- 
vais choix en venant se fixer et en continuant de vivre en si grand nombre dans l'Est de l'Ontario. D'autant plus qu'il s'agissait d'une région qui était fort loin d'égaler le Centre, le Sud-Ouest de la province et la région de Montréal pour la qualité de la terre. Pourtant, une comparaison entre celle-ci et l'Est ontarien démontre qu'en agissant ainsi, ces individus, en plus d'avoir accès à la terre, avaient certainement, d'une façon ou d'une autre, réussi à améliorer leur sort.

D'aucuns seront, peut-être, surpris de constater, en voyant ces chiffres, que la performance des agriculteurs de l'Est de l'Ontario était supérieure, dans l'ensemble, à celle de leurs voisins de la région de Montréal. La chose est d'autant plus intrigante que la qualité des terres dans la région de Montréal équivalait, non à celle de l'Est, mais à celle du Centre et du Sud-Ouest ontariens et que, dans le Centre, la production des grains par occupant doublait presque celle de l'Est. Comment, dans ces conditions, pourraiton, sans faire intervenir d'autres facteurs, continuer à expliquer les disparités entre le Québec et l'Ontario en invoquant seulement la qualité des terres?

\section{IV - LES CONCENTRATIONS AGRICOLES FRANCOPHONES}

Dans leur choix d'un lieu d'émigration, les Canadiens français des Maritimes et du Québec avaient done marqué une grande préférence pour certaines régions de l'Ontario, en particulier, pour l'Est et le Nord. Cette sorte d'attrait n'était pas nouveau, puisqu'à la même époque, le nord du Nouveau-Brunswick exerçait une attirance semblable sur les migrants des Maritimes. 
Mais ce mouvement ne s'arrêta pas là, puisqu'à l'intérieur de chaque région, en Ontario et ailleurs, il y eut des zones qui firent l'objet d'une égale préférence. Ainsi, les comtés d'Essex et Kent dans le SudOuest ontarien, celui de Simcoe dans le Centre et, dans l'Est, les quatre comtés les plus voisins du Québec, ceux de Prescott, Russell, Stormont et Glengarry, illustrent parfaitement ces tendances.

Ceci ne veut pourtant pas dire que les migrants d'origine française, bien que se regroupant à certains endroits, aient abandonné le reste du territoire aux autres. Car, loin de limiter leur quête d'emplois et de terres à un seul lieu, ils se répandirent jusqu'à un certain point sur l'ensemble du territoire. Déjà, au recensement de 1851 , la mention d'origine française apparaissait dans tous les comtés, sauf deux. Très vite, cependant, cette présence devint de plus en plus universelle, non seulement à ce niveau mais dans les moindres localités. Ainsi, en 1911, la liste des cités, villes et villages de toutes dimensions en Ontario incluait 278 agglomérations: seulement seize d'entre elles n'avaient aucun francophone parmi leurs habitants mais quarante et une d'entre elles en dénombraient de 1 à 20 . Donc, peu de place ici, même en situation de concentration, pour des communautés homogènes de langue française et de foi catholique. Il est vrai que, localement, à l'intérieur de certains comtés de l'est ontarien, tels Prescott et Russell, mais aussi, au Nouveau-Brunswick, à l'intérieur de Gloucester et Kent, la présence francophone dépassa souvent les $90 \%$ des habitants. Mais ailleurs, le caractère hétérogène des communautés locales, même situées les unes près des autres, fut, presque toujours, très marqué. Ce fut le cas à l'intérieur de Glengarry et 
Stormont dans l'Est ontarien, ainsi que dans Westmorland et Northumberland au Nouveau-Brunswick.

\section{LA CONCENTRATTON D'ESSEX-KENT}

Il va de soi que cette forte hétérogénéité existait dans Essex-Kent, les deux comtés les plus francophones du Sud-Ouest. Le peuplement canadienfrançais y était aussi ancien que le poste de Détroit établi par les Français au début du XVIII ${ }^{e}$ siècle et cédé aux Américains à la fin de leur Révolution. Vers 1800 , les colons anglophones avaient commencé à prendre des terres dans les cantons où les Canadiens français étaient en plus grand nombre. Vers 1850 , ces deux comtés, où la population devait tripler en soixante ans, ne contenaient que $17,7 \%$ de celle du Sud-Ouest. Par la suite, cette proportion déclina jusqu'en 1871 pour remonter à $21,2 \%$ en 1911 . La population rurale avait évolué de la même façon: de $17,1 \%$ de celle du Sud-Ouest en 1851 à $20 \%$ en 1911. Excepté pendant la décennie 1850-1860, alors que le nombre de Canadiens français avait diminué, leur présence parmi les ruraux de ces comtés ne varia pas tellement en cours de période: $19,6 \%$ en $1851,26,4 \%$ en 1881 et $18,8 \%$ en 1911 . Les francophones avaient, en gros, accru leurs effectifs au même rythme que les autres et, de cette façon, maintenu leur position minoritaire.

Le taux de concentration des Canadiens français dans ces comtés du Sud-Ouest était, au départ, très élevé. Car, en $1851,84,6 \%$ de leurs effectifs régionaux se trouvaient dans Essex-Kent. Bien que connaissant des bas au cours des décennies suivantes, cette proportion s'était d'un seul coup élevée de $75,7 \%$ 
en 1901 à $85,4 \%$ en 1911 . Les ruraux francophones s'étaient comportés de la même façon, à cette différence près que, pendant la dernière décennie de la période, leur taux de concentration était tombé à $66,6 \%$. Le tableau 24 illustre cette situation.

TABLEAU 24

Population d'Essex-Kent relativement au Sud-Ouest, 1851-1911: (1) Essex-Kent/Sud-Ouest, (2) Essex-Kent rural/Sud-Ouest rural, (3) Canadiens françals d'Essex-Kent/C.f. Sud-Ouest, (4) C.f. ruraux Essex-Kent/C.f. ruraux Sud-Ouest (en pourcentages)

\begin{tabular}{lllll}
\hline & $(1)$ & $(2)$ & $(3)$ & $(4)$ \\
1851 & 17,7 & 17,1 & 84,6 & 83,9 \\
1861 & 15,0 & 19,1 & 80,0 & 78,4 \\
1871 & 12,2 & 12,1 & 72,5 & 73,1 \\
1881 & 13,9 & 12,9 & 77,6 & 78,4 \\
1901 & 15,2 & 20,6 & 75,7 & 79,5 \\
1911 & 21,2 & 20,0 & 85,4 & 66,6 \\
\hline
\end{tabular}

SOURCES: Recensements du Canada.

En 1851, la colonisation agricole du Sud-Ouest, jusque-là en retard sur l'Est, était relancée. Ainsi, à cet endroit, s'amorçait une phase d'expansion démographique et économique rapide. Pendant la décennie suivante, la population s'accrut de $93 \%$ dans la région et de $64 \%$ dans Essex-Kent. Comme le potentiel agricole était probablement aussi bon dans un endroit que dans l'autre, les agriculteurs d'EssexKent suivirent le mouvement. Il est vrai que la production des grains et racines par occupant y fut jusqu'à la fin du siècle inférieure à ce qu'elle était dans le reste de la région; mais, à cette date, grâce aux énormes récoltes de maïs, ces deux comtés réalisèrent des performances supérieures à l'ensemble de 
la région. En 1901, la production de grains et racines par occupant surpassa les 1600 boisseaux dans Essex et les 1800 dans Kent, soit plus de deux fois la récolte du comté le plus productif du Québec. Dans le secteur de l'élevage, même sì celui du cheval, un élément de son avance passée dans ce domaine, avait régressé depuis 1851 , aussi bien que celui des bestiaux et des moutons, les agriculteurs de ces deux comtés s'assurèrent finalement une suprématie par l'importance unique qu'ils accordèrent au porc.

TABLEAU 25

La production des grains et racines et le cheptel par occupant dans

Essex-Kent comparés à ceux du Sud-Ouest, 1851-191 1

(en pourcentages)

\begin{tabular}{|c|c|c|c|c|c|c|}
\hline & blé & avoine & p. de terre & navets & \multicolumn{2}{|c|}{ grains et racines } \\
\hline 1851 & 90,6 & 84,0 & 92,9 & 33,3 & \multicolumn{2}{|r|}{98,0} \\
\hline 1861 & 41,4 & 86,7 & 193,0 & 18,9 & \multicolumn{2}{|r|}{71,4} \\
\hline 1871 & 82,9 & 80,8 & 73,1 & 12,9 & \multicolumn{2}{|r|}{86,0} \\
\hline 1881 & 92,7 & 65,7 & 67,2 & 3,6 & \multicolumn{2}{|r|}{77,5} \\
\hline 1901 & 78,4 & 81,6 & 80,2 & 12,4 & \multicolumn{2}{|c|}{123,5} \\
\hline 1911 & 115,1 & 79,5 & 72,1 & 1,0 & \multicolumn{2}{|c|}{112,5} \\
\hline & chevaux & vaches & b. à cornes & moutons & porcs & animaux \\
\hline 1851 & 153,3 & 108,0 & 101,4 & 102,3 & 119,6 & 107,6 \\
\hline 1861 & 140,9 & 100,0 & 94,7 & 93,3 & 151,5 & 113,7 \\
\hline 1871 & 129,6 & 82,8 & 82,1 & 89,4 & 150,9 & 105,1 \\
\hline 1881 & 110,7 & 78,4 & 78,5 & 49,3 & 178,0 & 91,0 \\
\hline 1901 & 105,9 & 79,5 & 75,2 & 59,6 & 206,7 & 118,1 \\
\hline 1911 & 107,7 & 73,2 & 66,9 & 84,4 & 183,9 & 115,8 \\
\hline
\end{tabular}

SOURCES: Recensements du Canada.

On peut se demander dans quelle mesure les $\mathrm{Ca}$ nadiens français de ces comtés participèrent à cette expansion. L'étude de Crothall ${ }^{19}$, couvrant les années ${ }^{19}$ W.R. Crothall, «French Agriculture in Ontario, 1861-1871», M.A. 
1861-1871, sur les fermiers du canton de SandwichOuest, à population majoritairement francophone, et sur ceux de Maidstone, canton peuplé d'une majorité anglophone, montre que les deux groupes étaient, pour le moment, en retard sur les localités du voisinage, notamment en ce qui regarde le volume de la récolte de blé. Les cultivateurs d'origine française y pratiquaient comme les autres une agriculture mixte; mais, étant donné le volume plus faible de leur production, ils étaient, semble-t-il, plus dépendants que leurs compatriotes du travail saisonnier en forêt. Assez rapidement, cependant, les deux communautés entrèrent dans le mouvement qui appelait un accroissement rapide et substantiel de la production. Les disparités entre les francophones et les anglophones qui habitaient Sandwich et Maidstone ne disparurent pas. Ainsi, en 1911 dans Sandwich-Est et Sandwich-Ouest, cantons francophones dans une proportion de plus de $70 \%$, la production de grains et racines par occupant s'élevait à environ 690 boisseaux contre une moyenne de 1200 dans Sandwich-Sud et Maidstone, canton à population anglophone dans une proportion d'environ 75\%. Dans Tilbury-Nord et Tilbury-Ouest, les mêmes disparités existaient mais les niveaux de production étaient beaucoup plus élevés dans les deux groupes. D'un autre côté, dans Rochester, canton à $60 \%$ francophone, les moyennes étaient, comme dans Camden, supérieures à celles de leurs comtés respectifs. Il est évident que le processus d'uniformisation des performances était en marche et que le niveau de production de ces fermiers surpassait par une marge considérable celui des francophones du Québec, de Simcoe et de l'Est de l'Ontario. Par contre, il se rapprochait

thesis, University of Toronto, 1968, $139 \mathrm{p}$. 
du niveau de ceux qui habitaient le comté de Prince sur l'île-du-Prince-Edouard, se tenant quand même en arrière de celui des producteurs établis au Manitoba et en Saskatchewan.

\section{LA CONCENTRATION DE SIMCOE}

Ces réalités émergent encore plus nettement dans le Centre, où le comté de Simcoe était le principal centre canadien-français. Celui-ci avait été formé à l'origine par un groupe de 160 coureurs des bois venus, en 1828, de l'île Drummond, en même temps qu'une trentaine de soldats britanniques. En 1851, ce comté comptait seulement 569 habitants d'origine

\section{TABLEAU 26}

Population de Simcoe relativement au Centre ontarien, 1851-1911*:

(1) Simcoe/Centre, (2) Simcoe rural/Centre rural, (3) Canadiens français de Simcoe/C.f. du Centre, (4) C.f. ruraux du Simcoe /C.f. ruraux du Centre (en pourcentages)

\begin{tabular}{rrrrr}
\hline & $(1)$ & $(2)$ & $(3)$ & $(4)$ \\
1851 & 6,1 & 6,9 & 10,2 & 11,6 \\
1861 & 7,0 & 8,0 & 20,7 & 22,9 \\
1871 & 8,1 & 9,6 & 24,4 & 31,1 \\
1881 & 12,5 & 12,3 & 25,5 & 29,9 \\
1901 & 14,4 & 14,5 & 30,6 & 43,5 \\
1911 & 14,6 & 14,1 & 32,9 & 50,1 \\
\hline
\end{tabular}

SOURCES: Recensements du Canada.

*Le comté de Simcoe avait fait l'objet d'une division nord/sud en 1871 , la concentration canadienne-française se trouvant alors dans SimcoeNord; en 1891, une nouvelle division avait eu lieu et la concentration francophone se trouvait maintenant dans Simcoe-Est.

française, dont 552 ruraux $(2,1 \%$ des ruraux du comté), qui représentaient seulement $10,2 \%$ des francophones du Centre. Soixante ans plus tard, Simcoe 
était le lieu de résidence de 6832 Canadiens français, dont 3828 étaient des ruraux. Donc, à la fin de ces soixante années de peuplement, $32,9 \%$ des francophones et $50,1 \%$ des ruraux de même origine ( $7,8 \%$ des ruraux du comté) du Centre habitaient dans Simcoe.

\section{TABLEAU 27}

Production agricole par occupant de (1) Simcoe-Nord/Centre de la province, (2) de Simcoe-Est/Centre de la province, (3) Simcoe-Nord/

Simcoe-Sud, (4) Simcoe-Est/Simcoe-Sud-Nord (en pourcentages)

\begin{tabular}{lrrrrrr}
\hline & $(1)$ & \multicolumn{1}{c}{$(2)$} & \multicolumn{1}{c}{$(2)$} & \multicolumn{1}{c}{$(3)$} & \multicolumn{1}{c}{$(4)$} & \multicolumn{1}{c}{$(4)$} \\
& 1881 & 1901 & 1911 & 1881 & 1901 & 1911 \\
grains et racines & 61,7 & 57,7 & 73,8 & 69,5 & 57,4 & 62,3 \\
blé & 71,6 & 60,0 & 40,4 & 41,0 & 39,4 & 18,9 \\
orge & 25,1 & 26,4 & 44,4 & 26,8 & 19,3 & 21,1 \\
avoine & 76,2 & 63,3 & 84,7 & 75,8 & 86,4 & 74,7 \\
p. de terre & 103,8 & 93,0 & 138,0 & 101,0 & 88,3 & 125,7 \\
navets & 42,3 & 67,0 & 40,4 & 129,5 & 67,0 & 51,8 \\
& & & & & & \\
animaux & 83,6 & 81,8 & 102,0 & 74,2 & 77,8 & 85,4 \\
chevaux & 78,1 & $\mathbf{7 5 , 7}$ & $\mathbf{8 8 , 8}$ & $\mathbf{7 3 , 5}$ & $\mathbf{7 5 , 7}$ & $\mathbf{7 4 , 4}$ \\
vaches & $\mathbf{8 8 , 2}$ & $\mathbf{8 2 , 5}$ & 95,1 & 83,3 & 97,0 & 105,4 \\
b. à cornes & $\mathbf{8 9 , 6}$ & $\mathbf{8 2 , 7}$ & 110,3 & 84,1 & 89,6 & 99,1 \\
moutons & 34,8 & 116,7 & 162,5 & 60,5 & 91,8 & 123,8 \\
porcs & 110,5 & 60,3 & 75,9 & 82,3 & 54,3 & 61,1 \\
& & & & & & \\
beurre & $\mathbf{7 7 , 2}$ & 94,8 & 137,6 & 77,7 & 96,3 & 114,3 \\
laine & 73,1 & 105,4 & 141,3 & 62,5 & 56,8 & 104,8
\end{tabular}

SOURCES: Recensements du Canada.

Simcoe était donc un lieu de modeste concentration francophone. Cependant, quand la population et la fraction rurale des trois comtés qui le composèrent après 1891 , commença à plafonner, voire à décliner, les Canadiens français et les ruraux de même origine continuèrent à s'accroître en nombre. Pour- 
tant, ce coin de terre du centre de la province, plus particulièrent la partie dense du peuplement francophone, était en dessous de la moyenne régionale pour sa production agricole. Le tableau 27 illustre ce fait pour Simcoe-Nord en 1881 et Simcoe-Est en 1901 et 1911 . Ces deux comtés avaient été créés successivement sur la portion du territoire habitée depuis le début par les francophones où $90 \%$ des francophones du comté originel résidaient maintenant.

Ceci dit, il faut bien se rendre compte que le niveau de production de Simcoe-Nord en 1881 et de SimcoeEst en 1901 et 1911 était bien supérieur à ce qu'il était aux mêmes dates dans les concentrations francophones de la Nouvelle-Écosse et du NouveauBrunswick. Elles étaient également beaucoup plus élevées qu'au Québec. Ainsi, pour les grains et racines par occupant: 609 boisseaux dans la concentration ontarienne francophone contre 392 au Québec en 1881,747 boisseaux contre 475 en 1901 et 761 contre 398 en 1911 . En 1881, 185 boisseaux dans la concentration francophone de la Nouvelle-Écosse et 368 dans celle du Nouveau-Brunswick. Les écarts, bien que moins marqués pour le cheptel et les produits domestiques, étaient quand même significatifs.

Ces migrants des Maritimes ou du Québec venus s'établir dans le centre de la province avaient certainement amélioré leur sort en quittant leur lieu d'origine. Néanmoins, en 1911, les disparités qui avaient existé entre eux et leurs voisins étaient encore considérables. Ainsi, en cette fin de période, la production de grains et racines dans six communautés rurales de Simcoe-Sud, ayant toutes moins de $1 \%$ de francophones, se situait entre 1300 et 1685 boisseaux. Dans Simcoe-Nord où la même situation prévalait à 
la même date, la récolte se chiffrait entre 864 et 1472 boisseaux. Dans la seule communauté rurale de Simcoe-Est, celle de Tiny, où les Canadiens français constituaient un pourcentage élevé de la population, soit $56,5 \%$, la récolte de grains et racines s'élevait à seulement 735 boisseaux, ce qui était quand même au-dessus de la moyenne québécoise. Dans Tay, avec $19,5 \%$ de francophones, la moyenne s'établissait à 705. Il faut dire que, dans ce comté, la meilleure performance appartenait aux habitants de Medante avec une production moyenne de 918 boisseaux.

\section{LA CONCENTRATION DE L'EST ONTARIEN}

La troisième concentration canadienne-française de l'Ontario se trouvait dans l'est de l'Est ontarien. En 1851, sa population était légèrement inférieure à celle d'Essex-Kent et onze fois plus considérable que celle de Simcoe. En 1911, elle était le double de la première et neuf fois le volume de la seconde. À cette date, sa population rurale quadruplait celle d'Essex-

\section{TABLEAU 28}

Population des quatre comtés orientaux de l'Est relativement à celle de l'Est ontarien, 1851-1911: (1) quatre comtés/Est, (2) effectifs ruraux: quatre comtés/est, (3) C.f. des quatre comtés/C.f. de l'Est, (4) C.f. ruraux des quatre comtés/C.f. ruraux de l'Est (en pourcentages)

\begin{tabular}{lccll}
\hline & $(1)$ & $(2)$ & $(3)$ & $(4)$ \\
1851 & 16,0 & 17,6 & 47,2 & 58,6 \\
1861 & 17,2 & 20,9 & 53,4 & 63,4 \\
1871 & 19,3 & 23,4 & 48,9 & 65,2 \\
1881 & 20,9 & 25,4 & 54,1 & 69,8 \\
1901 & 22,0 & 27,3 & 56,6 & 79,2 \\
1911 & 22,1 & 29,3 & 57,3 & 97,7 \\
\hline
\end{tabular}

SOURCES: Recensements du Canada. 
Kent. Fait original en Ontario, ses effectifs ruraux d'origine française étaient devenus majoritaires sur son propre territoire. Ils étaient seulement $14,5 \%$ des effectifs ruraux de ces quatre comtés en 1851 mais avaient atteint le niveau de $60,2 \%$ en 1911 . Le tableau 28 rend compte de l'évolution de ces rapports.

Incontestablement, le mouvement de concentration des Canadiens français dans ces quatre comtés s'apparente à celui qui avait lieu en même temps au Nouveau-Brunswick. Ce qui est remarquable dans les deux cas, c'est l'extraordinaire concentration progressive des effectifs canadiens-français, ruraux et autres, sur leur coin de territoire respectif. Cette présence acquit une telle intensité que c'est elle qui, peu à peu, détermina le profil agricole de ces régions. Le

\section{TABLEAU 29}

Production de grains et racines et le cheptel par occupant dans les quatre comtés orientaux de l'Est comparés à ceux de l'Est ontarien, 1851-1911 (en pourcentages)

\begin{tabular}{rrrrrcr}
\hline & blé & avoine & p. de terre & navets & grains et racines \\
1851 & 91,9 & 126,1 & 90,8 & 90,5 & 93,8 \\
1861 & 66,0 & 134,1 & 79,0 & 38,1 & 83,2 \\
1871 & 74,8 & 129,1 & 112,7 & 42,2 & 95,0 \\
1881 & 83,3 & 125,4 & 95,6 & 64,7 & 89,8 \\
1901 & 60,3 & 123,3 & 101,8 & 87,6 & 99,8 \\
1911 & 41,6 & 111,2 & 92,6 & 82,1 & 97,4 \\
& & & & & & \\
& chevaux & vaches & b. à comes & moutons & porcs & cheptel \\
1851 & 118,2 & 109,0 & 102,7 & 97,9 & 106,0 & 102,5 \\
1861 & 103,2 & 102,6 & 93,5 & 78,0 & 146,1 & 94,7 \\
1871 & 96,4 & 91,5 & 88,4 & 89,0 & 103,0 & 91,2 \\
1881 & 100,0 & 97,9 & 96,5 & 92,3 & 108,7 & 96,5 \\
1901 & 81,2 & 108,2 & 99,2 & 65,1 & 76,7 & 91,4 \\
1911 & 93,5 & 103,0 & 92,9 & 46,9 & 97,1 & 88,4 \\
& & & & & & \\
\hline
\end{tabular}

SOURCES: Recensements du Canada. 
tableau 29 sur l'agriculture dans la partie orientale de l'Est ontarien doit être comparé au tableau 23 qui met en rapport la région de Montréal et l'Est ontarien. Il apparaît alors que, non seulement l'Est ontarien mais aussi sa portion orientale, ellemême désavantagée par rapport à celui-ci, avait un avantage sur le district de Montréal.

Ce genre d'écart entre le territoire où étaient concentrés les francophones et la province se retrouve en Nouvelle-Écosse, mais il y semble plus cristallisé que partout ailleurs. La même observation vaut pour le nord du Nouveau-Brunswick relativement à la province.

Pour revenir aux quatre comtés de l'Est ontarien, l'écart qui existait entre eux et la région est incontestable, mais moins universel que celui observé dans les Maritimes. Car leur agriculture se rapprochait davantage, par l'importance accordée à la culture de l'avoine, de celle de l'Est ontarien et du district de Montréal, alors que celle du Nouveau-Brunswick francophone, par son attention à la pomme de terre, ressemblait plus à celle du district de Québec. Aussi, vers la fin du siècle, la marche vers l'industrie laitière y était-elle plus vigoureuse ici que là.

Cependant, il semble bien, si on isole Prescott, afin de voir les choses d'un peu plus près, que, dans le domaine de la production des grains et racines, loin de traîner derrière la région comme on s'y attendrait, ce comté eut souvent des performances supérieures à celle-ci. Pourtant, durant ces années, la proportion des Canadiens français dans Prescott était passée de $32,8 \%$ en 1851 à $74,6 \%$ en 1911 . Au niveau local, à l'intérieur de ce comté, la situation semble aussi plus complexe. À Alfred, par exemple, où $94 \%$ des 
habitants étaient francophones en 1911 , soit $20 \%$ de plus qu'en 1861, la production des grains et racines s'était accrue de 273 à 636 boisseaux par occupant. À Caledonia, au contraire, la récolte n'avait cessé de décliner à mesure que la population anglophone avait régressé de 80 à $31 \%$ de la population entre 1861 et 1911. La production de grains et racines par occupant y était passée de 563 boisseaux en 1861 à 322 en $1911^{20}$.

Notons, cependant, qu'en ce qui concerne l'élevage et les productions domestiques, les quatre comtés restaient à distance de l'ensemble de la région, sauf pour l'élevage du porc et, en fin de période, pour l'élevage des vaches laitières. Le fait est que, dans presque tous les coins de pays où se trouvaient des concentrations canadiennes-françaises, les cultivateurs avaient privilégié l'orientation vers l'industrie laitière et accordé assez peu d'attention aux animaux de boucherie relativement aux vaches laitières.

Il ne faudrait pas, simplement à cause de certaines améliorations et du tournant de l'industrie laitière, conclure à la prospérité relative des agriculteurs canadiens-francais du pays, de l'Ontario, y compris ceux qui résidaient sur le territoire des quatre comtés de l'Est ontarien. Le tableau 30 sur la valeur des fermes au Québec (la province la plus ancienne) et en Ontario en 1861 donne une certaine idée de l'ampleur et du caractère presqu'universel des inégalités qui affectent alors tous les groupes.

${ }^{20}$ Ouellet, dans C. Jaenen (éd.), Les Franco-Ontariens, op. cit., pp. 161, 171. 
TABLEAU 30

Valeur moyenne des fermes au Québec et en Ontario par régions en 1861 (en dollars)

\begin{tabular}{lrlrlr}
\hline Québec & & Ontario & & Est ontarien \\
province & 1904 & province & 2744 & quatre comtés & 1820 \\
Québec & 1379 & Sud-Ouest & 2095 & Prescott & 1375 \\
Trois-Rivières & 1481 & Centre & 3952 & Alfred (canton) & 510 \\
Montréal & 2360 & Est & 1908 & Caledonia (canton) 1 357 \\
& & Nord & 779 & &
\end{tabular}

SOURCE: Recensement de 1861.

Un autre son de cloche à ce sujet provient des listes électorales pour le comté de Prescott en 1883. Car, à cette époque, le suffrage censitaire existait encore, de sorte que ces listes reflétaient en gros le statut économique des groupes ethniques et sociaux en présence. À cette date, les francophones composaient $64 \%$ de la population mais ne fournissaient que $52 \%$ de ceux qui avaient le droit de vote ${ }^{21}$. Comme le taux d'urbanisation du comté était très faible, les disparités observées valaient surtout pour la classe des propriétaires de terres. Ceci dit, on doit quand même admettre que la position des francophones s'est améliorée progressivement jusqu'au $\mathrm{XX}^{\mathrm{e}}$ siècle et que les disparités entre eux et les autres ont été réduites d'autant.

\section{LA MARCHE VERS LE NORD}

La marche vers le Nord s'est accomplie selon deux directions principales: l'une partant de l'Est ontarien et l'autre du Centre et Sud-Ouest. La route venant de l'Est fut celle qu'empruntèrent davantage les migrants

${ }^{21}$ Chad Gaffield, Aux origines de l'identité franco-ontarienne. Éducation, culture, économie, (Ottawa, PUO, 1993), p. 177. 
d'origine française du Québec et de l'Est ontarien. Cette route qui attira aussi des anglophones, fut celle de la colonisation agricole stimulée par l'expansion de l'exploitation forestière, la construction des chemins et le développement minier.

La seconde, aux origines multiples, fut celle que suivirent plus volontiers un grand nombre d'anglophones qui émigrèrent vers le nord. Admettons que l'idée du Nord était fort relative dans les années 1860 , puisqu'à cette époque, même les territoires de la Muskoka et d'Owen Sound étaient si éloignés qu'ils attiraient peu de colons. Si lointains encore, qu'il faut suivre le sentiment des contemporains et les rattacher temporairement, pour la période 1851 à 1911 , à cette partie de la province. En vérité, cette route vers le nord devint facile d'accès seulement au moment de la construction, complétée en 1890, d'une voie ferrée de Toronto à North-Bay. Et ce ne fut pas avant 1908 que ce chemin de fer fut en mesure de traverser la région minière, donnant ainsi accès à la ceinture d'argile.

Cette avance des migrants francophones vers le nord était, donc, une progression vers des terroirs plus pauvres que ceux de l'Est de la province. L'agriculture qui s'y édifiait comportait une dépendance très marquée à l'égard du travail saisonnier en forêt. Cette structure, notons-le, n'était pas tout à fait différente de celle de l'Est, ni même de celle d'un grand nombre d'endroits au Québec et, à plus forte raison, des Maritimes où la pêche, la construction navale et l'exploitation forestière pouvaient se combiner aisément avec l'activité agricole.

Dans le Nord, l'agriculture, mixte comme ailleurs, reposait sur l'avoine, la pomme de terre et les navets, 
avec un peu de blé, denrées qui constituaient le plus gros de la récolte. La mise en place de cette structure se fit assez rapidement, puisqu'en 1881 , la production de grains et racines par occupant $y$ égalait presque celle de la Nouvelle-Écosse: 264 contre 267 boisseaux. Vingt ans plus tard, elle la doublait. Évidemment, en 1881, le cheptel était encore peu important: sept animaux seulement par occupant. Mais, en 1901 , ces effectifs avaient été augmentés à dix-sept. Dix ans plus tard, les Canadiens français étaient concentrés dans Algoma-Est et dans Nipissing dans une proportion de $31,9 \%$ et leurs performances surpassaient même la moyenne de la région. Disons, cependant, qu'ils étaient loin derrière les quatre comtés de l'Est oriental et de la région de Montréal.

\section{Conclusion}

Cette analyse, disons-le immédiatement, ne va pas assez loin dans sa description de la situation exacte de chaque communauté agraire francophone en milieu ontarien. En ce sens, elle n'a que valeur d'étude préliminaire. Par contre, la démarche géographique, temporelle et comparative adoptée, parce qu'elle est axée sur la reconstitution de grands ensembles et se meut, en mouvements successifs, vers les plus petits espaces, est celle qui permet le mieux de dégager le contexte et la structure soumis au test du temps.

En effet, cette façon de faire a permis, entre autres, de clarifier certains points de l'historiographie relatifs à la soi-disant transition du blé à l'industrie laitière. Car historiens et économistes, à cause de leur grande préoccupation pour le modèle des «staples», ont assumé que partout, à l'origine, les fermiers canadiens avaient eu tendance à pratiquer une agricul- 
ture basée sur la culture du blé et que l'émergence d'un système mixte aurait été une réponse au déclin universel du blé sur les terroirs. La vérité est que, excepté dans le Québec d'avant 1800, l'agriculture mixte a partout existé à l'origine: dans les Maritimes, en Ontario et même dans l'Ouest avant 1911.

De tout temps, dans les Maritimes avant le XIX ${ }^{\mathrm{e}}$ siècle, le poisson étant la base de l'alimentation et l'agriculture une activité secondaire combinée à d'autres activités, le blé n'eut qu'une importance marginale dans les récoltes. Pas étonnant que cette région ait toujours été importatrice de farine et qu'elle continuât de le faire durant la seconde moitié du $\mathrm{XIX}^{\mathrm{e}}$ siècle. En Ontario, même si le blé trouva progressivement un marché extérieur et devint un "staple», il ne fut, à l'origine et par la suite, qu'un élément parmi d'autres dans une agriculture mixte qui devint de plus en plus diversifiée. Son déclin, mesuré en termes absolus, qui se produisit bien avant que l'Ouest n'entrât en scène, signifiait éventuellement sa chute en tant que "staple" mais ne changeait aucunement le caractère mixte de l'agriculture. Dans l'Ouest, en Colombie-Britannique surtout, mais aussi au Manitoba, en Saskatchewan et en Alberta, l'activité agricole fut établie, au départ, sur des cultures variées et ne changea de caractère, lorsqu'elle le fit, qu'au fil des décennies. Dans ces conditions, comment peut-on continuer à lier le développement de l'industrie laitière, phénomène qui se produit partout, à la désintégration d'une structure agricole basée essentiellement sur le blé?

À cet égard, seul le Québec d'avant 1800 marqua son originalité en adoptant en tous lieux le blé comme production dominante: entre 65 et $75 \%$ de la récolte 
du XVII ${ }^{e}$ siècle jusqu'au début du XIX ${ }^{\mathrm{e}}$ siècle. En effet, le pain y était une des bases essentielles de l'alimentation et le blé servait, en partie, au paiement de la dîme, de la Quête de l'Enfant-Jésus, des redevances seigneuriales et, même, des achats chez le marchand. Avec le temps, il devint aussi une denrée commerciale vendue à l'intérieur et à l'extérieur: donc, jusqu'à un certain point, un «staple». En 1831, son déclin en tant que culture dominante et «staple» était un fait accompli. Au Québec seul, on pourrait peut-être lier la marche vers l'industrie laitière au blé: (1) si le déclin du blé ne s'était opéré longtemps avant la mise en place de cette industrie; (2) si, traditionnellement, les cultivateurs canadiensfrançais avaient accordé une importance minimum à la fabrication du beurre et du fromage domestiques; (3) et si les premiers responsables de la fabrication sur la ferme des produits laitiers, les anglophones des cantons, avaient cultivé le blé autrement que d'une façon marginale. Pour comprendre ces changements, il faut donc chercher ailleurs, notamment du côté des rapports entre industrialisation, urbanisation et déclin général des productions domestiques.

Notre démarche a aussi permis de montrer comment, après 1851 , cette agriculture mixte, devenue une pratique universelle sur le territoire, une fois le Québec entré dans le cercle, s'est diversifiée, à vrai dire, plus facilement à certains endroits qu'à d'autres. Il faut donc reconnaître, à l'arrière-plan, l'action de forces qui jouèrent en tous lieux dans cette direction mais qui eurent des effets variés selon les régions, les sous-régions et les moments. L'émergence de l'industrie laitière apparaîtrait aussi, en un sens, comme une expression généralisée, à un moment donné et à des degrés divers, de ce processus de diversification. 
Ainsi, d'une façon universelle en 1851 , qu'il s'agisse des Maritimes, du Québec et de l'Ontario, régnait une agriculture mixte qui, dès le départ, fut aussi celle des colons francophones qui s'établirent dans les différentes régions et localités de l'Ontario. Cependant, dans la plupart des endroits, le Québec inclus, où les Canadiens français étaient concentrés, le blé, l'avoine et la pomme de terre accaparaient un tel pourcentage de la récolte qu'il y restait peu de place pour une diversification aisée des cultures. Il y eut, bien sûr, des exceptions: Digby-Richmond en 1871 et 1881 en Nouvelle-Écosse, en Ontario, EssexKent à partir de 1871 et Simcoe en 1851 seulement, et, au Manitoba, Provencher à partir de 1881. Bien qu'un aspect original de l'agriculture de ces territoires, cette concentration intense du travail autour de quelques cultures n'empêcha pas, à un moment donné, les francophones d'aller avec plus de résolution que d'autres vers l'industrie laitière.

Ainsi, la démarche qui consiste à mettre en évidence des similarités dans les structures et les tendances est sans doute fondamentale puisqu'elle met l'accent sur des destins communs et des solidarités. Pourtant, tel que le démontre aussi cette évolution, l'univers des vivants est aussi un lieu habité par une multitude de différences et d'inégalités, reflétant aussi bien l'interaction de forces géographiques et économiques que de facteurs démographiques et culturels.

En envisageant les structures et les sources de changement sous cet angle de la diversité, il est apparu en même temps que là où les agriculteurs canadiens-français étaient les plus concentrés sur le territoire canadien, le niveau de production des grains et racines était, d'une façon significative, beaucoup 
plus bas qu'ailleurs. En $1851,92,5 \%$ de la population rurale francophone était regroupée au Québec et dans les Maritimes, mais, sur ces terroirs, la production de grains et racines par habitant de la campagne n'était que de 52 boisseaux contre 105 à l'ouest du Québec. En 1911, le pourcentage de la population rurale canadienne-française vivant dans ces provinces de l'Est était tombé à $84 \%$. Mais l'écart, en ce qui concerne la production des grains et racines, s'était élargi considérablement: 60 boisseaux dans la zone Québec-Maritimes contre 191 à l'ouest du Québec.

Il est encore plus surprenant de constater qu'à l'intérieur même du Québec, le même genre de disparités existait depuis l'époque française. En 1851, les Canadiens français ruraux étaient sur-représentés dans les districts de Québec et Trois-Rivières: $49 \%$ des ruraux francophones s'y trouvaient et ne récoltaient que $43 \%$ des grains et racines. Là aussi, en 1911 , le fossé s'était élargi puisque $62,8 \%$ des ruraux d'origine française y vivaient et ne produisaient que $48 \%$ des grains et racines de la province.

Le même type de situation s'était reconstitué en Ontario où les Canadiens français étaient surtout concentrés dans les régions les moins productives de la province: l'Est et le Nord. En 1851 et 1911, seulement un tiers de la population rurale de la province résidait dans ces deux parties de la province. Les ruraux canadiens-français qui, au début, y avaient $48 \%$ de leurs effectifs provinciaux, accrurent la densité de leur présence à $75 \%$. Pourtant, ces régions perdirent du terrain, surtout, en ce qui concerne la production des grains et racines par rapport à celle de la province: $24 \%$ en 1851 et $19 \%$ en 1911 . Bien 
que moins prononcées dans le secteur de l'élevage des animaux, ces disparités n'en étaient pas moins réelles.

Ces performances inégales dans les grandes cultures se retrouvaient aussi à l'intérieur même des régions où les migrants canadiens-français avaient créé des concentrations. Quelques exceptions, cependant: une fois dans le nord du Nouveau-Brunswick en 1871 et une seconde dans Prince-Albert-Moosejaw en Alberta en 1911. En Ontario, la concentration d'Essex-Kent devint pour de bon, semble-t-il, une exception en 1901. Naturellement, si l'enquête était davantage poussée au niveau des comtés individuels et des localités, les nuances se trouveraient encore multipliées.

On serait peut-être porté, comme on l'a fait si souvent, à attribuer les écarts Québec-Ontario aux seuls facteurs géographiques et à la qualité des sols. Si tel était le cas, les producteurs agricoles de l'Est ontarien, y compris les Canadiens français du lieu, auraient souffert d'une énorme infériorité par rapport à ceux de la région de Montréal, fortement avantagée par la qualité des sols. Pourtant, si surprenant que cela soit, qu'il s'agisse de la production des grains ou du nombre d'animaux par occupant, il faut reconnaître que l'Est ontarien et les quatre comtés de la concentration francophone de cette région eurent, dans l'ensemble, de meilleures performances que celles de la région de Montréal.

Quoi qu'il en soit des causes de ces inégalités, on ne doit pas oublier que ces différences reflétaient des disparités de revenus et des chances plus limitées quant à l'accumulation des capitaux. Mais, à cet égard, les inégalités n'étaient pas qu'ethniques, elles 
étaient aussi sociales. Des auteurs comme Gwyn, Siddiq, Inwood et Wagg ont posé le problème mais en s'intéressant seulement aux disparités entre les fermiers et le reste de la population. C'est aussi la préoccupation de Livio Di Matteo et de Peter George dans leur étude du comté de Wentworth en Ontario, 1872-1902, qui concluent:

The evidence presented in this paper demonstrates that, as in other areas of North America during the later nineteenth century, wealth was very unequally distributed in Wentwoth county. The question, of course, is why, despite the great inequality that marked late nineteenthcentury society there was not more popular protest against such unequality, and even social upheavel? ${ }^{22}$

Aucun de ces auteurs n'a tenté d'utiliser ses sources pour savoir dans quelle mesure la classe agricole elle-même était habitée par les inégalités. Peut-être ont-ils eux-mêmes cru, comme beaucoup d'autres, à l'égalitarisme paysan! Un peu comme si, à l'origine, la terre avait été distribuée également; comme si, en plus, la mesure fort relative d'égalité, qui a pu exister au départ, aurait pu, si elle a existé, résister à tous les changements démographiques, économiques et institutionnels subséquents. En 1872, beaucoup de chemin avait été parcouru. D'ailleurs, la constitution de ce dossier de l'inégalitarisme paysan, amorcée il y a quelques décennies et mis de côté plus récemment, a quand même produit des résultats assez substantiels.

Marvin McGinnis prétend à cet égard qu'en 1861 , un sixième des fermes ontariennes n'avait aucun surplus écoulable sur le marché. Mais il est égale-

${ }^{22}$ Livio Di Matteo and Peter George, "Canadian Wealth Inequality in the Late Nineteenth Century: A Study of Wentworth County, Ontario, 1872-1902", Canadian Historical Review, LXXIII, 1992, p. 482. 
ment important de savoir quel était ce pourcentage dans les différentes régions de la province. Lorsqu'on sait que la production moyenne et le cheptel moyen par occupant, estimés à 781 boisseaux et à 26 animaux dans la province, étaient de 1069 boisseaux et 30 bêtes dans le Centre, 679 et 24 dans le SudOuest, 531 et 24 dans l'Est, 337 et 16 dans le Nord? Qu'en était-il au Québec où, à la même date, la production moyenne et le cheptel moyen étaient seulement de 422 boisseaux et de 19 animaux? D'ailleurs, la diversité régionale y était non moins présente que dans la province voisine. Les chiffres pour les régions étaient: 362 boisseaux et 18 animaux pour Québec, 404 et 17 pour Trois-Rivières, 466 et 21 pour Montréal. Ainsi, d'un lieu à un autre et d'un groupe ethnique à un autre selon leur degré de concentration sur le terroir, cette proportion peut même passer d'un sixième à un tiers ou davantage. Tout ceci apporte de l'eau au moulin d'une historiographie qui a du chemin à faire et dans laquelle le FrancoOntarien, qui n'a pas reçu sa part d'attention, ne saurait être perçu exclusivement ni comme un travailleur isolé, ni comme un être communautaire. 